Title

\title{
Correlation of mitochondrial TOM core complex stop-and-go and open-closed channel dynamics
}

\author{
Authors \\ Shuo Wang ${ }^{1}$, Lukas Findeisen ${ }^{1}$, Sebastian Leptihn ${ }^{2}$, Mark I. Wallace ${ }^{3}$, Marcel Hörning ${ }^{4, *}$, \\ Stephan Nussberger ${ }^{1, *}$
}

\author{
Affiliations \\ ${ }^{1}$ Department of Biophysics, Institute of Biomaterials and Biomolecular Systems, \\ University of Stuttgart, Pfaffenwaldring 57, 70569 Stuttgart, Germany. \\ ${ }^{2}$ Zhejiang University-University of Edinburgh (ZJU-UoE) Institute, Zhejiang University, \\ 718 East Haizhou Rd., Haining, Zhejiang 314400, China. \\ ${ }^{3}$ Department of Chemistry, King's College London, Britannia House, 7 Trinity Street, \\ London, United Kingdom. \\ ${ }^{4}$ Department of Biobased Materials, Institute of Biomaterials and Biomolecular Systems, \\ University of Stuttgart, Pfaffenwaldring 57, 70569 Stuttgart, Germany. \\ *Corresponding authors. Email: Stephan.Nussberger@bio.uni-stuttgart.de, \\ Marcel.Hoerning@bio.uni-stuttgart.de
}

\begin{abstract}
The role of lateral diffusion of proteins in the membrane in the context of function has not been examined extensively. Here, we explore the relationship between protein lateral diffusion and channel activity of the general protein import pore of mitochondria (TOM-CC). Optical ion flux sensing through single TOM-CC molecules shows that TOM-CC can occupy three ion permeability states. Whereas freely diffusing TOM-CC molecules are preferentially found in a high permeability state, physical tethering to an agarose support causes the channels to transition to intermediate and low permeability states. This data shows that combinatorial opening and closing of the two pores of TOM-CC correlates with lateral protein diffusion in the membrane plane, and that the complex has mechanosensitive-like properties. This is the first demonstration of $\beta$-barrel protein mechanosensitivity, and has direct conceptual consequences for the understanding of the process of mitochondrial protein import. Our approach provides a novel tool to simultaneously study the interplay of membrane protein diffusion and channel dynamics.
\end{abstract}




\section{Introduction}

The TOM complex of the outer membrane of mitochondria is the main entry gate for nuclearencoded proteins from the cytosol into mitochondria (Wiedemann and Pfanner, 2017). It does not act as an independent entity, but in a network of interacting protein complexes, which transiently cluster in mitochondrial outer- and inner-membrane contact sites (Pfanner et al., 2019; Scorrano et al., 2019; Wurm et al., 2011). Recent studies suggest a functional crosstalk between TOM and chaperones, which guide preproteins from the cytosol to mitochondria (Becker et al., 2019; Namba, 2019), cytosolic ribosomes (Gold et al., 2017), and the endoplasmic reticulum (Becker et al., 2019; Namba, 2019). For proteins destined for integration into the lipid bilayer of the inner mitochondrial membrane, TOM transiently cooperates with components of the inner membrane protein translocase TIM22. Proteins en route to the mitochondrial matrix require supercomplex formation with the inner membrane protein translocase TIM23 (Chacinska et al., 2005; Gold et al., 2014; Mokranjac et al., 2009). Thus, depending on the activity of mitochondria, the lateral mobility of TOM within the mitochondrial outer membrane may be fundamental to the different import needs of the organelle (Pfanner et al., 2019). The relationship between the mode of lateral mobility (Jacobson et al., 2019) and TOM protein function, however, has not yet been explored. In particular, it is important to know whether the main entry gate for nuclear-encoded proteins is open or closed when associated with structures at the membrane periphery, as this could have direct conceptual implications for mitochondrial protein import.

Detailed insights into the molecular architectures of the TOM core complex (TOM-CC) from $N$. crassa (Bausewein et al., 2017), S. cerevisiae (Araiso et al., 2019; Tucker and Park, 2019) and human (Wang et al., 2020) mitochondria have been obtained by cryo-electron microscopy (cryoEM). All three structures show well-conserved symmetrical dimers, where the monomer comprises five membrane protein subunits. Each of the two transmembrane $\beta$-barrel domains of the protein-conducting subunit Tom40 interacts with one subunit of Tom5, Tom6, and Tom7, respectively. Two central transmembrane Tom 22 receptor proteins, reaching out into the cytosol and the mitochondrial inter membrane space (IMS), connect the two Tom40 pores at the dimer interface. The path of polypeptides through theses pores is considered identical to the path of ions through the complex.

Previous studies reported that the TOM complex channels from wild-type yeast mitochondria are mainly in closed state (van Wilpe et al., 1999). However, TOM isolated from mutant tom $22 \Delta$ mitochondria without Tom22 was mainly found in open state (van Wilpe et al., 1999), resembling that observed with purified Tom40 (Ahting et al., 2001; Hill et al., 1998). This indicated that the "multifunctional organizer" Tom 22 of the mitochondrial protein translocase negatively regulates the open probability of the Tom40 channels of the TOM-CC (van Wilpe et al., 1999). Nevertheless, the molecular mechanism of how Tom22 affects the open-closed channel activity of the TOM machinery has remained an open question.

Most studies that reported on the dynamic and functional properties of the TOM-CC channel have been based on ion current measurements through single TOM-CC channels in planar lipid membranes under application of a membrane potential $\left(\Delta \psi_{\mathrm{m}}\right)$ (Ahting et al., 1999; Becker et al., 2005; Hill et al., 1998; Künkele et al., 1998; Kuszak et al., 2015; Mager et al., 2011; Mahendran et al., 2013). TOM-CC has been found to switch between a complex set of conductance states under this experimental setting. Tom 22 has been proposed to contribute additional flexibility to the complex by reducing the energy required for transitions between these states (Poynor et al., 2008; Romero-Ruiz et al., 2010). However, the physiological significance of these voltagedependent transitions has been controversially discussed because the critical voltage above which 
TOM-CC channels close is significantly larger than any possible Donnan potential $\left(\Delta \psi_{\text {Don }}\right)$ across the outer mitochondrial membrane (Benz et al., 1990; Lemeshko and Lemeshko, 2000; Lemeshko, 2002).

In this work, we simultaneously probe and correlate the lateral mobility and the ion flux though single TOM-CC molecules by means of non-invasive Electrode-free Optical Single Channel Recording (Ef-oSCR) (Demuro and Parker, 2005; Huang et al., 2015; Leptihn et al., 2013; Wang et al., 2018; Yuqin Wang et al., 2019). Possible changes in ion flux associated with conformational changes of the TOM-CC are only caused by thermal fluctuations and the nature of lateral diffusion of the TOM-CC in the membrane. This leads to new insights into the open-closed dynamics of the TOM-CC channel and the corresponding nature of lateral movement, which are difficult to illuminate by voltage-clamp, biochemical assays or structural analysis (Davis et al., 2021).

We find that freely diffusing TOM-CC molecules stall when interacting with structures adjacent to the membrane, ostensibly due to interaction between the extended polar domains of Tom 22 and a supporting agarose layer. Concomitantly, with transient or permanent suspension of movement, the TOM-CC switches from active (both pores open) to weakly active (one pore open) and inactive (both pores closed) states.

The strong temporal correlation between lateral mobility and channel activity suggests that TOM$\mathrm{CC}$ function is highly sensitive to lateral diffusion and mechanical constraints. Taken alongside recent cryo-electron microscopy of this enigmatic complex, we argue that these dynamics provide a new functionality of the TOM-CC that has not been anticipated before. From a general perspective, for the best of our knowledge this is the first demonstration of $\beta$-barrel protein mechanoregulation and the causal effect of lateral diffusion. So far, mechanosensitivity has only been observed in primarily $\alpha$-helical protein channels. The applied experimental approach can be easily transferred to other systems, where channel activity additionally to lateral membrane diffusion is of importance.

\section{Results}

\section{Visualizing the open-closed channel activity of TOM-CC}

Owing to the unresolved complexity of the mitochondrial outer membrane, TOM-CC was isolated from a $N$. crassa strain, that carries a version of subunit Tom 22 with a hexahistidine tag at the Cterminus (Fig. 1A), and reconstituted into a well-defined supported lipid membrane (Kiessling et al., 2015; Sackmann, 1996; Tanaka and Sackmann, 2005). Droplet interface membranes (DIBs) were created through contact of lipid monolayer-coated aqueous droplets in a lipid/oil phase and a lipid monolayer on top of an agarose hydrogel (Huang et al., 2015; Leptihn et al., 2013; Wang et al., 2018; Yuqin Wang et al., 2019). The cis side of the membrane contained $\mathrm{Ca}^{2+}$-ions, while having a $\mathrm{Ca}^{2+}$-sensitive fluorescent dye (Fluo-8) at the trans side. $\mathrm{Ca}^{2+}$-ion flux through individual TOM-CCs was measured by monitoring Fluo- 8 emission in close proximity to the membrane using TIRF microscopy in the absence of membrane potential (Ef-oSCR) to avoid voltagedependent TOM-CC gating (Figs. 1B and 1C).

Contrary to the classical single molecule tracking approach using single fluorescently labeled proteins, we have an almost instantaneous update of fluorophores close to the TOM-CC nanopores. This enables a spatiotemporal tracking of individual molecules with much higher accuracy and longer observation time up to a couple of minutes. 
Upon TIRF illumination of membranes with $488 \mathrm{~nm}$ laser light, single TOM-CC molecules appeared as high-contrast fluorescent spots on a dark background (Fig. 2A). High $\left(\mathrm{S}_{\mathrm{H}}\right)$, intermediate $\left(\mathrm{S}_{\mathrm{I}}\right)$ and low $\left(\mathrm{S}_{\mathrm{L}}\right)$ intensity levels were indicating $\mathrm{Ca}^{2+}$-flux through the TOM-CC in three distinct permeability states. No high-contrast fluorescent spots were observed in membranes without TOM-CC. The fact that the TOM-CC is a dimer with two identical $\beta$-barrel pores (Fig. 1B) suggests that the high and intermediate intensity levels correspond to two conformational states $\left(\mathrm{S}_{\mathrm{H}}\right.$ and $\left.\mathrm{S}_{\mathrm{I}}\right)$ with two pores and one pore open, respectively. The low intensity level may represent a conformation $\left(\mathrm{S}_{\mathrm{L}}\right)$ with both pores closed.

Movie S1 shows an optical recording of the open-closed channel activity of several TOM-CCs over time. Individual image frames of membranes were recorded at high frequency at a frame rate of $47.5 \mathrm{~s}^{-1}$ and corrected for fluorescence bleaching. The position and amplitude of individual spots were determined by fitting their intensity profiles to a two-dimensional symmetric Gaussian function with planar tilt to account for possible local illumination gradients in the bleaching corrected background (Fig. 2B and Movie S2). The time evolution of amplitude signals (Fig. 2C and Movie S2) shows that the TOM-CC does not occupy only one of the permeability states $\mathrm{S}_{\mathrm{H}}$, $S_{I}$ and $S_{L}$, but can switch between these three permeability states over time (Fig. 2D). Additional examples are shown in Fig. S1. These data allow us to conclude that the fluctuations between the three defined permeability states are an inherent property of TOM-CC.

To rule out the possibility that the observed intensity fluctuations are caused by possible thermodynamical membrane undulations in the evanescent TIRF illumination field (Duncan et al., 2017), or by local variations in $\mathrm{Ca}^{2+}$ flux from cis to trans (Wang et al., 2018; Yuqin Wang et al., 2019), we visualized the ion flux through a three-pore $\beta$-barrel which is almost entirely embedded in the lipid bilayer (Figs. S2A and S2B). In a series of control experiments, we reconstituted E. coli OmpF (Benz, 2006) into DIB membranes and observed virtually constant fluorescence intensities (Figs. 2E and 2F, and Figs. S2C - S2F). The protein did not exhibit any gating transitions between its four different permeability states (one to three pores open, all pores closed) (Benz, 2006), which normally require voltage to induce closure. The toggling of the TOM$\mathrm{CC}$ between the three different permeability states $\mathrm{S}_{\mathrm{H}}, \mathrm{S}_{\mathrm{I}}$ and $\mathrm{S}_{\mathrm{L}}$ (Fig. 2C) therefore had to have another cause.

\section{The permeability states of TOM-CC are coupled to lateral mobility}

Since the functions of many integral membrane proteins depend on their local position and state of movement in the membrane (Davis et al., 2021; Fujiwara et al., 2016; Jacobson et al., 2019; Koppel et al., 1981), we attempted to capture individual TOM-CCs in one of the permeability states $\mathrm{S}_{\mathrm{H}}, \mathrm{S}_{\mathrm{I}}$ or $\mathrm{S}_{\mathrm{L}}$. To this end, we simultaneously tracked the open-closed activity and position of individual TOM-CC molecules in the membrane over time using the same experimental conditions as described above. A scheme of the experiment is shown in Fig. 3A.

Movie S3 and Fig. 3B clearly show that the open-closed channel activity of single TOM-CCs is coupled to lateral movement in the membrane. This is supported by comparison of the trajectories of single TOM-CC molecules with their corresponding fluorescence amplitude traces (Figs. 3C and 3D). The position of fluorescent spots does not change when TOM-CC is in intermediate $S_{I}$ or low $\mathrm{S}_{\mathrm{L}}$ permeability state. Although weak intensity profiles do not allow accurate determination of the position of TOM-CC in the membrane plane, Movie S3 and Fig. 3D clearly show that TOM$\mathrm{CC}$ does not move in $\mathrm{S}_{\mathrm{L}}$; disappearance and reappearance of the fluorescent spot, switching from $S_{I}$ to $S_{L}$ and back to $S_{I}$, occurs at virtually the same spatial $x, y$-coordinates. In contrast, the trajectories of TOM-CC in $\mathrm{S}_{\mathrm{H}}$ state demonstrate free diffusion. Additional samples of trajectories and amplitude traces are shown in Fig. S1. 
Similar stop-and-go movement patterns were observed in an independent set of experiments for TOM-CC covalently labeled with fluorescent dye Cy3 (Movie S4 and Fig. S3). It is particularly striking that freely moving TOM-CC molecules stop at the same spatial x,y-position when they cross the same position a second time, indicating a specific molecular trap or anchor point at this stop-position below the membrane. OmpF, however, used as a control, shows the most elementary mode of mobility expected for homogeneous membranes: simple Brownian translational diffusion (Movie S5 and Fig. S2). Since OmpF is almost entirely embedded in the membrane, there seems to be no coupling between channel activity and lateral protein diffusion.

In good agreement with these results, the diffusion coefficients of the TOM-CC, evaluated from the activity profiles and trajectories (Fig. 3 and Fig. S1), were determined from time-averaged mean squared displacements as $D_{\mathrm{I}}=D_{\mathrm{L}} \leq D_{\min }=0.01 \mu^{2} \mathrm{~s}^{-1}$ and $D_{\mathrm{H}} \simeq 0.85 \pm 0.16$ (mean $\pm S E M$, $n=46)$ in states $\mathrm{S}_{\mathrm{I}}$ and $\mathrm{S}_{\mathrm{H}}$, respectively. TOM-CC molecules, that revealed a diffusion constant less or equal than $D_{\min }$, were defined as immobilized. The diffusion coefficient $D_{\mathrm{H}}$ corresponds to typical values of Tom40 $\left(D_{\mathrm{Tom} 40} \sim 0.5 \mu \mathrm{m}^{2} \mathrm{~s}^{-1}\right)$ and Tom7 $\left(D_{\operatorname{Tom} 7} \sim 0.7 \mu \mathrm{m}^{2} \mathrm{~s}^{-1}\right)$ in native mitochondrial membranes (Kuzmenko et al., 2011; Sukhorukov et al., 2010) and is comparable to that of transmembrane proteins in plasma membranes lined by cytoskeletal networks $(39,43)$. The diffusion coefficient of TOM-CC in $\mathrm{S}_{\mathrm{L}}$ state could not always be reliably determined here due to its extremely low intensity levels (see Materials and Methods).

TOM-CC labeled with Cy3 yielded diffusion constants of $D_{\mathrm{Cy} 3, \mathrm{H}} \simeq 0.36 \pm 0.08 \mu \mathrm{m}^{2} \mathrm{~s}^{-1}$ (mean \pm SEM, $n=15$ ) and $D_{\mathrm{Cy} 3, \mathrm{I}} \leq D_{\min }=0.01 \mu \mathrm{m}^{2} \mathrm{~s}^{-1}$ for moving and transiently trapped particles, respectively (Fig. S3). In line with typical values for protein in homogenous lipid membranes (Koppel et al., 1981; Ramadurai et al., 2009), the lateral diffusion coefficient of the control protein OmpF was determined as $D_{\mathrm{OmpF}} \simeq 1.16 \pm 0.07 \mu \mathrm{m}^{2} \mathrm{~s}^{-1}$ (mean $\pm \mathrm{SEM}, n=42$ ) (Fig. S2).

Based on these results we conclude that transient arrest of TOM-CC in a lipid bilayer membrane, caused by interaction with the supporting hydrogel, triggers the closure of its two $\beta$-barrel pores. Since retarded diffusion of membrane proteins is largely the result of cluster formation of proteins near membrane surfaces (Nawrocki et al., 2019), this phenomenon is important for studying preprotein trapping at either Tom $40 \beta$-barrel when TOM-CC interacts with proteins at the periphery of the outer membrane.

\section{Controlled immobilization of the TOM-CC results in channel closures}

Because the two Tom 22 subunits in the middle of the TOM-CC clearly protrude from the membrane plane at their IMS side (Fig. 1B) (Araiso et al., 2019; Bausewein et al., 2017; Tucker and Park, 2019; Wang et al., 2020), we hypothesized that Tom22 functions as a "light-switch" and determines the lateral mobility of the TOM-CC and thus the transitions between open $\left(\mathrm{S}_{\mathrm{H}}\right)$ and the closed $\left(\mathrm{S}_{\mathrm{I}}\right.$ and $\left.\mathrm{S}_{\mathrm{L}}\right)$ conformations of the two TOM-CC pores.

To test this hypothesis, we replaced the non-modified agarose hydrogel with Ni-NTA-modified agarose to further restrict lateral movement of the protein by permanently tethering individual TOM-CC molecules via the C-terminus of histidine-labeled Tom22 to the hydrogel (Fig. 4A).

In good agreement with the observation that transient closure of the two Tom40 $\beta$-barrels accompanies transient arrest of the TOM-CC (Figs. 3C and 3D), permanently immobilized TOM$\mathrm{CC}\left(D_{\mathrm{I}, \mathrm{L}} \leq D_{\min }=0.01 \mu \mathrm{m}^{2} \mathrm{~s}^{-1}, n=83\right)$ was most often found in states $\mathrm{S}_{\mathrm{I}}$ or $\mathrm{S}_{\mathrm{L}}$, indicating one or two pores closed, respectively (Movie S6 and Fig. 4B, Fig. S4A - S4D). TOM-CC molecules that were not immobilized by the binding of Tom 22 to Ni-NTA moved randomly in the membrane plane $\left(D_{\mathrm{H}} \simeq 0.34 \pm 0.06 \mu \mathrm{m}^{2} \mathrm{~s}^{-1}\right.$, mean $\left.\pm S E M, n=40\right)$. As expected, movement was interrupted 
occasionally by periods of transient arrest $\left(D_{\mathrm{I}, \mathrm{L}} \leq D_{\min }\right)$. Again, moving TOM-CC molecules were found in the fully open $S_{H}$ state; non-moving complexes in the $S_{I}$ or the $S_{L}$ states (Fig. 4C; Figs. $\mathrm{S} 4 \mathrm{E}-\mathrm{S} 4 \mathrm{H})$. In the presence of imidazole, which prevents tight binding of histidine-tagged Tom 22 to Ni-NTA-modified agarose, virtually no permanently immobilized TOM-CC molecules were observed $\left(D_{\mathrm{H}} \simeq 1.35 \pm 0.14 \mu \mathrm{m}^{2} \mathrm{~s}^{-1}\right.$, mean $\left.\pm S E M, n=15\right)$.

\section{Correlation between lateral motion and TOM-CC channel activity}

DIB membranes supported by both hydrogels, non-modified and Ni-NTA-modified agarose (Movie S6), showed a statistically significant number of single TOM-CC molecules that were either non-diffusive $D_{\mathrm{H}, \mathrm{I}} \leq D_{\min }$ or diffusive $D_{\mathrm{H}}>D_{\min }$ at $\mathrm{S}_{\mathrm{I}}$ and $\mathrm{S}_{\mathrm{H}}$. Thus, TOM-CC molecules were numerically sorted into diffusive and permanently tethered groups by $D_{\mathrm{H}}$ and $D_{\mathrm{I}}$ to emphasis the correlation between the mode of lateral diffusion and channel activity of 187 observed TOMCC molecules (Fig. 5A). We can generally define three different classes of lateral motion and channel activity. The first and major class (I) shows lateral mobility at $\mathrm{S}_{\mathrm{H}}$ only, while being tethered at $\mathrm{S}_{\mathrm{I}}\left(D_{\mathrm{H}}>D_{\min }\right.$ and $\left.D_{\mathrm{I}} \leq D_{\min }\right)$. The second class (II) shows similar diffusivities $\left(D_{\mathrm{H}, \mathrm{I}}>\right.$

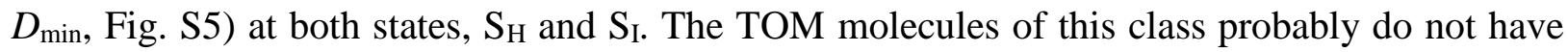
functional Tom22 (Ahting et al., 2001; Shiota et al., 2015) and thus do not interact with the hydrogel. Another possible but unlikely explanation is a spatial void of agarose network preventing mechanoregulated interaction of Tom 22 with the network within the observation time window. The third class (III) represents permanently tethered TOM-CC molecules, which are exclusively non-diffusive $\left(D_{\mathrm{H}, \mathrm{I}} \leq D_{\mathrm{min}}\right)$. Most molecules of that class are in $\mathrm{S}_{\mathrm{I}}$ and $\mathrm{S}_{\mathrm{L}}$ (Figs. S4A and S4B). Those TOM-CC molecules, which briefly change from $S_{I}$ to $S_{H}$ and back to $S_{I}$ (Figs. S4C and S4D), might become diffusive but are immediately recaptured and trapped again by the hydrogel below the membrane.

Fig. 5B shows state probabilities of TOM-CC in membranes supported by the two different hydrogels, non-modified and Ni-NTA-modified agarose. Diffusive molecules $\left(D_{\mathrm{H}}>D_{\min }\right)$ in membranes supported both by non-modified and Ni-NTA-modified agarose show similar probabilities to be at one of the three permeability states $\left(\mathrm{S}_{\mathrm{H}}, \mathrm{S}_{\mathrm{I}}\right.$ and $\left.\mathrm{S}_{\mathrm{L}}\right)$. Diffusive TOM-CC molecules are significantly more often at $S_{H}$ than at $S_{I}$ and $S_{L}$. The permanently tethered fraction of TOM-CC $(67 \%)$ in Ni-NTA-modified agarose is $~ 2.4$ times larger compared to the fraction $(28 \%)$ in non-modified agarose, consistent with the stronger interaction of Tom 22 with the hydrogel, thereby permanently constraining lateral motion. In line with this, permanently tethered molecules $\left(D_{\mathrm{H}, \mathrm{I}} \leq D_{\mathrm{min}}\right)$ in both hydrogel-supported membranes stay at $\mathrm{S}_{\mathrm{I}}$ during the majority of time, and show only transient $S_{H}$ and $S_{L}$ occupancy. The data suggest that the C-terminal IMS domain of Tom22 (Fig. 1B) plays a previously unrecognized role in mechanoregulation of TOM$\mathrm{CC}$ channel activity by binding to immobile structures near the membrane.

Although diffusive TOM-CC molecules $\left(D_{\mathrm{H}}>D_{\min }\right)$ are observed more often at $\mathrm{S}_{\mathrm{H}}$ in Ni-NTAmodified agarose than in non-modified agarose supported membranes (Fig. 5B), they show a lower stability at $\mathrm{S}_{\mathrm{H}}$ having a significantly higher transition probability for switching between $\mathrm{S}_{\mathrm{H}}$ and $\mathrm{S}_{\mathrm{I}}\left(\right.$ Fig. $5 \mathrm{C},\left(\mathrm{S}_{\mathrm{H}} \leftrightarrows \mathrm{S}_{\mathrm{I}}\right) \simeq 5.3 \%$ versus $\left.\left(\mathrm{S}_{\mathrm{H}} \leftrightarrows \mathrm{S}_{\mathrm{I}}\right) \simeq 2.3 \%\right)$. This is in line with the higher efficacy of TOM-CC-trapping by Ni-NTA-modified agarose compared to non-modified agarose. In contrast to non-modified hydrogel, Ni-NTA-modified agarose hydrogel can capture freely mobile TOM-CC via the IMS domain of Tom 22 in two ways: by specific interaction and permanent anchorage with Ni-NTA and by nonspecific collision and transient anchorage. While a direct transition between $S_{H}$ and $S_{L}$ barely occurs in both systems, transitions between $S_{I}$ and $S_{L}$ are similarly often. This indicates that the two Tom $40 \beta$-barrel pores independently open and close within the time resolution ( $20 \mathrm{~ms})$ of our experiment. 


\section{Discussion}

TOM-CC is usually considered as a passive conduit for mitochondrial preproteins and small molecules across the outer mitochondrial membrane, which is regulated by interactions with other proteins and posttranslational modifications (Rapaport et al., 1998; Schmidt et al., 2011).

In this study, we have shown for the first time that lateral diffusion of isolated TOM-CC in the plane of the membrane is coupled to transmembrane channel activity. Conversely, restriction of the lateral mobility of the complex by the surrounding matrix influences channel activity in a defined manner. This in turn indicates that TOM-CC does not only respond to biochemical (Rapaport et al., 1998; Schmidt et al., 2011), but also to yet-unknown mechanical stimuli: anchorage of freely moving TOM-CC to structures near the membrane leads to TOM-CC channel closing.

Our conclusions are based upon data obtained from single molecules, which yield insights into molecular events that are difficult to obtain in vivo and in bulk methods. Typically, single molecule methods are often subject to statistical uncertainties arising from the limited amount of measurements, which are technically feasible. For this reason, we have obtained data from $n=$ 187 single molecules and over half a million image frames (Fig. 5A, $N=532,576$ ). This sampling number allows us to assign molecular events with statistical confidence using non-parametric statistics, but even a visual perusal of the data is sufficient to allow a first assessment of data reliability. Our data indicate three molecular states of the TOM-CC, for which the simplest model is a dimeric pore structure with one or two pores open and all pores closed. As indicated above, the state transitions are responding to interaction with the supporting agarose matrix. We believe this mechanoregulated interactive effect, as we observed, is of physiological relevance.

Previous super-resolution fluorescence and immuno-electron microscopy studies have revealed a dynamic submitochondrial distribution of the protein translocation machineries TOM and TIM23 in the outer and inner membrane of mitochondria, both of which depend on the physiological state of the cell (Palmer et al., 2021; Vogel et al., 2006; Wurm et al., 2011). An attractive possibility would be that interaction of the TOM complex with subunits of TIM23 in the mitochondrial intermembrane space (IMS) via their subunits Tom22 and Tim50, respectively, might be coordinated so that protein import through the TOM channels can only occur when TIM23 is locally available (Callegari et al., 2020; Chacinska et al., 2005; Mokranjac et al., 2009; van der Laan et al., 2006). Thus, in the light of transient cooperation between TOM and TIM23 (Donzeau et al., 2000; Gevorkyan-Airapetov et al., 2009; Waegemann et al., 2015) it is becoming increasingly clear that the interplay between lateral diffusion and channel activity of these complexes is pivotal for protein translocation into mitochondria.

However, previous studies beg a fundamental question: are the two Tom40 channels open continually, as seems to be the case from the static cryoEM structures (Fig. 1B; (Araiso et al., 2019; Bausewein et al., 2017; Tucker and Park, 2019; Wang et al., 2020), or can the protein channels be actively influenced by interaction with exogenous, interacting proteins? Previous studies reported that TOM complex channels from wild-type yeast mitochondria were mainly in the closed state (van Wilpe et al., 1999). However, TOM isolated from mitochondria lacking Tom22 was mainly in the open state (van Wilpe et al., 1999).

In our study, we have demonstrated that Tom 22 plays an important role in the kinetics of TOMCC channel activation. Examination of the cryoEM structure of $N$. crassa TOM-CC (Fig. 1B, (Bausewein et al., 2020, 2017)) shows that its two Tom22 subunits extend significantly into the 
IMS space, thereby preventing a direct interaction of the Tom $40 \beta$-barrels with the agarose matrix. It is therefore likely that only the two Tom 22 subunits are primarily responsible for the matrixdependent mechanoregulated channel closing activity. First, this hypothesis is supported by our observation that His-tagged Tom22, when tightly bound by Ni-NTA-agarose, triggers channel closing (Movie S6). Secondly, the cryoEM structure of TOM-CC reveals that the dimer interface of the Tom40 barrels is extremely limited, with only nine amino acid residues contributing to the interface at the cytosolic side of the barrel (Araiso et al., 2019; Bausewein et al., 2020, 2017; Tucker and Park, 2019; Wang et al., 2020). At the IMS side of the barrel, two conserved lysine residues (Tom40-K298, contributed from each Nc. crassa Tom40 monomer) seem to be close enough to severely destabilize the dimer. In fact, most of the stabilizing interactions of the Tom40 dimer seem to be contributed by residues from the interacting, transmembrane $\alpha$-helices of Tom 22 and a lipid phosphate group (Araiso et al., 2019; Bausewein et al., 2020; Tucker and Park, 2019). Thus, it seems reasonable that the conformation of the Tom40 barrel dimer is essentially determined by the positioning of two Tom 22 subunits. Taken together, it is tempting to suggest that, in our experiments, when the intermembrane space (IMS)-located polar domains of the Tom 22 subunits (which extend more than $22 \AA$ into the IMS, Fig. 1B) interact with the matrix of the agarose gel, the Tom 22 subunits force the Tom 40 dimer interface to experience a conformational change resulting in channel closure.

From a mechanistic point of view, this might be caused by classic rigid body rotation at the Tom 40 dimeric interface, perpendicular to the membrane normal. Rigid body rotation at subunit interfaces is a common feature of quaternary conformational changes in oligomeric proteins, for which the archetypical paradigm is rotation of the $\alpha_{1} \beta_{1}-\alpha_{2} \beta_{2}$ interface of hemoglobin during the oxy-deoxy allosteric transition (Baldwin and Chothia, 1979). The Tom40 dimer rigid body rotation might allow the disordered 84 amino acid residues, cytosolically located N-terminal domain of the Tom 22 subunit, which is not visible in the cryoEM structures, to "fall into" the Tom40 channel, thereby blocking $\mathrm{Ca}^{2+}$ export in the TIRF experiment. In vivo, this conformational change might be caused by tethering the IMS domain of Tom22 to Tim50 of the TIM23 machinery, which is virtually immobile in the mitochondrial inner boundary membrane $\left(D_{\operatorname{Tim} 23} \simeq 0.04 \mu \mathrm{m}^{2} \mathrm{~s}^{-1}\right)$ (Albrecht et al., 2006; Appelhans and Busch, 2017; Callegari et al., 2020; Chacinska et al., 2005; Mokranjac et al., 2009; van der Laan et al., 2006).

Previous studies suggested that a dimeric Tom22-free form of Tom40 dynamically exchanges with a trimeric TOM complex in outer mitochondrial membranes (Araiso et al., 2019; Shiota et al., 2015). In this study, we have not explicitly considered the functional relevance of TOM trimers. First, all of the cryoEM data obtained for detergent-solubilized TOM-CCs published so far (Araiso et al., 2019; Bausewein et al., 2017; Tucker and Park, 2019; Wang et al., 2020), indicates the dimer as the major subunit form. Secondly, we do not require a trimer to interpret the channel activity observed here. In fact, the functional trimer would require a fourth channel state, which has rarely been observed. Finally, the formation of trimers from functional dimers seems to be at variance with well-known principles of protein symmetry at subunit interfaces. Therefore, in lieu of more biophysical data from defined systems, we presently do consider the dimer and not the trimer of Tom40 to be the major functional form of the TOM-CC.

While eukaryotic secretion systems allow passage through a single bilayer, proteins that enter organelles of endosymbiotic origin must pass through two bilayers and translocation machineries to reach the interior of the organelle (Davis et al., 2021). Thus, future approaches should consider the multiprotein nature of the TOM-TIM import system, and how this additional layer of complexity is affected by the role of mechanoregulation in native mitochondrial membranes with TOM subunits Tom 20 and Tom70 in the presence of mitochondrial preproteins (Wiedemann and 
Pfanner, 2017). However, since Tom 20 and Tom70 do not have appreciable IMS domains and both proteins are only loosely bound to the complex (Ahting et al., 1999; Wiedemann and Pfanner, 2017), it seems unlikely that these proteins significantly influence the interplay between lateral diffusion and channel kinetics of the TOM-CC. The diffusion coefficient of mobile TOM-CC shown in our work is in good agreement to that of mobile Tom40 in native mitochondria (Kuzmenko et al., 2011; Sukhorukov et al., 2010). We therefore propose that the stop-and-go concept described here also applies to native mitochondria, in which proteins can freely move only on smaller length scales. TOM-CC channels may simply be trapped more frequently, leading to a higher frequency of open-closed transitions.

Recently, the now extensive structural and physical data common to mechanosensitive membrane proteins have been reviewed (Haswell et al., 2011; Jin et al., 2020; Kefauver et al., 2020). The structural data now allows mechanosensitive proteins (all of which were comprised of transmembrane $\alpha$-helices) to be separated into five different classes, each subject to characteristic underlying molecular mechanisms. They also show that for the membrane channels considered, fundamental physical properties of the membrane can influence channel activity. This ancient mechanism to regulate open-closed channel activity is also observed in mitochondria (Deng et al., 2020; Lee et al., 2016; Li et al., 2020; Walewska et al., 2018).

Mechanosensitivity via force transmission from a tether to the extracellular matrix and/or the cytoskeleton has been established for a number of $\alpha$-helical membrane proteins (Brohawn et al., 2014a, 2014b; Ge et al., 2018; Jin et al., 2020; Kefauver et al., 2020; Martinac, 2004; Pliotas and Naismith, 2017; Teng et al., 2015; Yang Wang et al., 2019). These findings go along with our observations for the TOM-CC, which seems to obey the "tether model" proposed by Kefauver et al. (2020) (Kefauver et al., 2020), where an intermembrane protein anchor domain limits lateral diffusion. To our knowledge, the TOM-CC complex would thus be the only example so far, of a membrane $\beta$-barrel protein, which exhibits membrane state-dependent mechanosensitive-like properties.

\section{Materials and Methods}

Growth of Neurospora crassa and preparation of mitochondria

Neurospora crassa (strain GR-107) that contains a hexahistidinyl-tagged form of Tom22 was grown and mitochondria were isolated as described (Künkele et al., 1998). Briefly, $\sim 1.5 \mathrm{~kg}$ (wet weight) of hyphae were homogenized in $250 \mathrm{mM}$ sucrose, $2 \mathrm{mM}$ EDTA, $20 \mathrm{mM}$ Tris $\mathrm{pH} 8.5,1$ $\mathrm{mM}$ phenylmethylsulfonyl fluoride (PMSF) in a Waring blender at $4^{\circ} \mathrm{C} . \sim 1.5 \mathrm{~kg}$ of quartz sand was added and the cell walls were disrupted by passing the suspension through a corundum stone mill. Cellular residues were pelleted and discarded in two centrifugation steps $(4,000 \mathrm{xg})$ for 5 min at $4^{\circ} \mathrm{C}$. The mitochondria were sedimented in $250 \mathrm{mM}$ sucrose, $2 \mathrm{mM}$ EDTA, $20 \mathrm{mM}$ Tris $\mathrm{pH} 8.5,1 \mathrm{mM}$ PMSF at 17,000 $\mathrm{x}$ g for $80 \mathrm{~min}$. This step was repeated to improve the purity. The isolated mitochondria were suspended in $250 \mathrm{mM}$ sucrose, $20 \mathrm{mM}$ Tris $\mathrm{pH} 8.5,1 \mathrm{mM}$ PMSF at a final protein concentration of $50 \mathrm{mg} \mathrm{ml}^{-1}$, shock-frozen in liquid nitrogen and stored at $-20^{\circ} \mathrm{C}$.

\section{Isolation of TOM core complex}

TOM-CC, containing subunits Tom40, Tom22, Tom7, Tom6 and Tom5, were purified from Neurospora crassa strain GR-107 as described (Bausewein et al., 2017). N. crassa mitochondria were solubilized at a protein concentration of $10 \mathrm{mg} / \mathrm{ml}$ in $1 \%(\mathrm{w} / \mathrm{v}) \mathrm{n}$-dodecyl- $\beta$-D-maltoside (Glycon Biochemicals, Germany), 20\% (v/v) glycerol, $300 \mathrm{mM} \mathrm{NaCl}, 20 \mathrm{mM}$ imidazole, $20 \mathrm{mM}$ Tris- $\mathrm{HCl}(\mathrm{pH} 8.5)$, and $1 \mathrm{mM}$ PMSF. After centrifugation at 130,000 x g, the clarified extract was 
loaded onto a nickel-nitrilotriacetic acid column (Cytiva, Germany). The column was rinsed with the same buffer containing $0.1 \%$ (w/v) n-dodecyl- $\beta$-D-maltoside and TOM core complex was eluted with buffer containing $0.1 \%$ (w/v) n-dodecyl- $\beta$-D-maltoside, $10 \%$ (w/v) glycerol, $20 \mathrm{mM}$ Tris (pH 8.5), $1 \mathrm{mM} \mathrm{PMSF}$, and $300 \mathrm{mM}$ imidazole. For further purification, TOM core complex containing fractions were pooled and loaded onto a Resource $\mathrm{Q}$ anion exchange column (Cytiva) equilibrated with $20 \mathrm{mM}$ Hepes ( $\mathrm{pH} 7.2$ ), $2 \%$ (v/v) dimethyl sulfoxide and $0.1 \%$ (w/v) n-dodecyl$\beta$-D-maltoside. TOM core complex was eluted with $0-500 \mathrm{mM} \mathrm{KCl}$. A few preparations contained additional phosphate $(\sim 0.19 \mathrm{mM})$. The purity of protein samples $(0.4-1.2 \mathrm{mg} / \mathrm{ml})$ was assessed by sodium dodecyl sulfate polyacrylamide gel electrophoresis (SDS-PAGE) followed by staining with Coomassie Brilliant Blue.

\section{Fluorescence labeling of TOM core complex}

TOM-CC was covalently labeled with the fluorescent dye Cy3 according to (Joo and Ha, 2008). Briefly, about $1 \mathrm{mg} / \mathrm{ml}$ of purified TOM-CC was reacted with Cy3-maleimide (AAT Bioquest, USA) at a molar ratio complex to dye of 1:5 in $20 \mathrm{mM}$ HEPES ( $\mathrm{pH} 7.2), 2 \%$ (v/v) dimethyl sulfoxide, $350 \mathrm{mM} \mathrm{KCl}$ and $0.1 \%$ (w/v) n-dodecyl- $\beta$-D-maltoside at $25^{\circ} \mathrm{C}$ for $2 \mathrm{~h}$ in the dark. Labeled protein was separated from unconjugated dye by affinity chromatography using Ni-NTA resin, subjected to SDS-PAGE and visualized by $555 \mathrm{~nm}$ light and Coomassie Brilliant Blue staining.

\section{Isolation of OmpF}

Native OmpF protein was purified from Escherichia coli strain BE BL21(DE3)omp6, lacking both LamB and OmpC as described (Bieligmeyer et al., 2016). Cells from $1 \mathrm{~L}$ culture were suspended in $50 \mathrm{mM}$ Tris-HCl, pH 7.5 buffer containing $2 \mathrm{mM} \mathrm{MgCl}_{2}$ and DNAse and broken by passing through a French press. Unbroken cells were removed by a low-speed centrifugation, then, the supernatant was centrifuged at 100,000 x g for $1 \mathrm{~h}$. The pellet was resuspended in 50 $\mathrm{mM}$ Tris- $\mathrm{HCl}, \mathrm{pH} 7.5$, and mixed with an equal volume of SDS buffer containing $4 \%(\mathrm{w} / \mathrm{v})$ sodium dodecyl sulfate (SDS), $2 \mathrm{mM} \beta$-mercaptoethanol and $50 \mathrm{mM}$ Tris- $\mathrm{HCl}, \mathrm{pH} 7.5$. After 30 min incubation at a temperature of $50{ }^{\circ} \mathrm{C}$, the solution was centrifuged at 100,000 $\mathrm{g}$ for $1 \mathrm{~h}$. The pellet was suspended in $2 \%(\mathrm{w} / \mathrm{v}) \mathrm{SDS}, 0.5 \mathrm{M} \mathrm{NaCl}, 50 \mathrm{mM}$ Tris- $\mathrm{HCl}, \mathrm{pH} 7.5$, incubated at a temperature of $37{ }^{\circ} \mathrm{C}$ for $30 \mathrm{~min}$ and centrifuged again at $100,000 \mathrm{x} \mathrm{g}$ for $30 \mathrm{~min}$. The supernatant containing OmpF was dialyzed overnight against $20 \mathrm{mM}$ Tris, $\mathrm{pH} 8,1 \mathrm{mM}$ EDTA and $1 \%$ (w/v) n-octyl polyoxyethylene (Octyl-POE, Bachem, Switzerland). The purity of the protein was assessed by SDS-PAGE.

\section{Formation of droplet interface bilayers}

Droplet interface bilayer (DIB) membranes were prepared as previously described (Huang et al., 2015; Leptihn et al., 2013; Wang et al., 2018; Yuqin Wang et al., 2019) with minor modifications. Glass coverslips were washed in an ultrasonic bath with acetone. Then the coverslips were rinsed several times with deionized water and dried under a stream of nitrogen. Subsequently, the glass coverslips were subjected to plasma cleaning for $5 \mathrm{~min}$. $140 \mu \mathrm{l}$ of molten $0.75 \%$ (w/v) low melting non-modified agarose $\left(\mathrm{T}_{\mathrm{m}}<65^{\circ} \mathrm{C}\right.$, Sigma-Aldrich) or alternatively low melting Ni-NTA-modified agarose (Cube Biotech, Germany) was spin-coated at 5,000 rpm for $30 \mathrm{~s}$ onto the plasma-cleaned side of a glass coverslip. After assembly of the coverslip in a custom-built DIB device, the hydrogel film was hydrated with $2.5 \%$ (w/v) low melting agarose, $0.66 \mathrm{M} \mathrm{CaCl}_{2}$ and $8.8 \mathrm{mM}$ HEPES (pH 7.2) or with $2.5 \%$ (w/v) low melting agarose, $0.66 \mathrm{M} \mathrm{CaCl}_{2}, 300 \mathrm{mM}$ imidazole and $8.8 \mathrm{mM}$ HEPES ( $\mathrm{pH} \mathrm{7.2)}$ and covered with a lipid/oil solution containing $9.5 \mathrm{mg} / \mathrm{ml} \mathrm{1,2-}$ diphytanoyl-sn-glycero-3-phosphocholine (DPhPC, Avanti Polar Lipids, USA) and 1:1 (v:v) mixture oil of hexadecane (Sigma-Aldrich) and silicon oil (Sigma-Aldrich). Aqueous droplets $(\sim 200 \mathrm{nl})$ containing $7 \mu \mathrm{M}$ Fluo-8 sodium salt with a maximum excitation wavelength of $495 \mathrm{~nm}$ 
(Santa Cruz Biotechnology, USA), $400 \mu \mathrm{M}$ EDTA, $8.8 \mathrm{mM}$ HEPES (pH 7.2), 1.32 M KCl, 2.7 $\mathrm{nM}$ TOM core complex or $\sim 2 \mathrm{nM} \mathrm{OmpF}$ were pipetted into the same lipid/oil solution in a separate tray using a Nanoliter 2000 injector (World Precision Instruments, Sarasota, USA). After 2 hours of equilibration at room temperature, the droplets were transferred into the custom-built DIB device to form stable lipid bilayers between the droplet and the agarose hydrogel.

\section{TIRF microscopy and optical recording}

An inverted total internal reflection fluorescence microscope (Ti-E Nikon) was used to image DIB membranes under TIRF illumination using a $488 \mathrm{~nm}$ laser (Visitron). Fluorescent emission of Fluo-8, transmitted through a Quad-Band TIRF-Filter (446/523/600/677 HC, AHF), was collected through a $100 \mathrm{x}$ oil objective lens (Apochromat N.A. 1.49, Nikon) and recorded by a backilluminated electron-multiplying CCD camera (iXon Ultra 897, $512 \times 512$ pixels, Andor) for 1 $\min$ at a frame rate of $47.51 \mathrm{~s}^{-1}$. The pixel size was $0.16 \mu \mathrm{m}$.

\section{Tracking of fluorescence spots}

For reliable tracking and analysis of the spatiotemporal dynamics of individual fluorescence TOM-CC channel activity a customized fully automated analysis routine was implemented in Matlab (The Mathworks, USA). The effect of bleaching was corrected by considering a double exponential decay obtained by least square fitting of the image series. No filter algorithm was applied. The initial spatial position of a fluorescence spot was manually selected and within a defined region of interested (ROI, 30 x 30 pixels) fitted to a two-dimensional symmetric Gaussian function with planar tilt that accounts for possible local illumination gradients, as follows

$$
G_{2 D}\left(\boldsymbol{x}, \boldsymbol{\mu}, p_{k}\right)=p_{1}+p_{2,3}(\boldsymbol{x}-\boldsymbol{\mu})+A \exp \left(-(\boldsymbol{x}-\boldsymbol{\mu})^{2} / 2 \sigma^{2}\right)
$$

where $\boldsymbol{x}=(x, y)$ is the ROI with the fluorescence intensity information, A and $\sigma$ are the amplitude and width of the Gaussian, $p_{k}$ are parameters that characterize the background intensity of the ROI, and $\boldsymbol{\mu}=\left(x_{0}, y_{0}\right)$ defines the position of the Gaussian. The latter was used to update the position of ROI for the next image. Spots that temporal fuse their fluorescence signal with closely located spots were not considered due to the risk of confusing those spots.

\section{Data analysis}

The extracted amplitudes were separated by two individually selected amplitude-thresholds that dived the three states of activity $\left(\mathrm{S}_{\mathrm{H}}, \mathrm{S}_{\mathrm{I}}\right.$ and $\left.\mathrm{S}_{\mathrm{L}}\right)$. The lateral diffusion constants $D_{\mathrm{H}}$ and $D_{\mathrm{I}}$ were obtained individually for spots within the respective high and intermediate amplitude range by linear regression of the time delay $\tau$ and the mean square displacement of the spots. Further details may be found in SI Experimental Methods. As

$$
D=\frac{\left\langle\left|\boldsymbol{r}(x, y, \tau)-\boldsymbol{r}\left(x_{0}, y_{0}, \tau\right)\right|^{2}\right\rangle}{4 \tau}
$$

The largest time delay $\tau_{\max }=0.5 \mathrm{~s}$ was iteratively decreased to suffice the coefficient of determination $R^{2} \geq 0.9$ for avoiding the influence of sub-diffusion or insufficient amount of data. This followed the general approach of a Brownian particle in two-dimensions. The diffusions less than $D_{\min }=0.01 \mu \mathrm{m}^{2} \mathrm{~s}^{-1}$ are defined as non-diffusive considering the spatiotemporal limitations of the experimental setup and resolution of the fitted two-dimensional Gaussian function, since most led to $R^{2}<<0.9$. The calculation of the mean $\mu$ and standard deviation $\sigma$ of the diffusion constant was done using the log-transformation due to its skewed normal distribution, as 


$$
\mu_{\log }=\frac{1}{N} \sum_{i=1}^{N} \log \left(D_{i}\right)
$$

$$
\sigma_{\log }=\sqrt{\frac{1}{N} \sum_{i=1}^{N}\left(\log \left(D_{i}\right)-\mu_{\log }\right)^{2}}
$$

where $N$ is the number of diffusion constants obtained for one experimental condition. The backtransformation was calculated then as

$$
\mu=\exp \left(\mu_{\log }+0.5 \cdot \sigma_{\log }^{2}\right)
$$

And

$$
\sigma=\sqrt{\mu^{2}\left(\exp \left(\sigma_{\log }^{2}\right)-1\right)}
$$

respectively, following the Finney estimator approach (Finney, 1941). The standard error of mean (SEM) considering a confidence interval of $95 \%$ was calculated as

$$
S E M=\frac{1.96 \sigma}{\sqrt{N}}
$$

\section{References}

Ahting U, Thieffry M, Engelhardt H, Hegerl R, Neupert W, Nussberger S. 2001. Tom40, the poreforming component of the protein-conducting TOM channel in the outer membrane of mitochondria. J Cell Biol 153:1151-1160. doi:10.1083/jcb.153.6.1151

Ahting U, Thun C, Hegerl R, Typke D, Nargang FE, Neupert W, Nussberger S. 1999. The TOM core complex: the general protein import pore of the outer membrane of mitochondria. $J$ Cell Biol 147:959-968. doi:10.1083/jcb.147.5.959.

Albrecht R, Rehling P, Chacinska A, Brix J, Cadamuro SA, Volkmer R, Guiard B, Pfanner N, Zeth K. 2006. The Tim 21 binding domain connects the preprotein translocases of both mitochondrial membranes. EMBO Rep 7:1233-1238. doi:10.1038/sj.embor.7400828

Appelhans T, Busch KB. 2017. Dynamic imaging of mitochondrial membrane proteins in specific suborganelle membrane locations. Biophys Rev 9:345-352. doi:10.1007/s12551-017-0287-1

Araiso Y, Tsutsumi A, Qiu J, Imai K, Shiota T, Song J, Lindau C, Wenz L-S, Sakaue H, Yunoki K, Kawano S, Suzuki J, Wischnewski M, Schütze C, Ariyama H, Ando T, Becker T, Lithgow T, Wiedemann N, Pfanner N, Kikkawa M, Endo T. 2019. Structure of the mitochondrial import gate reveals distinct preprotein paths. Nature 575:395-401. doi:10.1038/s41586-019-1680-7

Baldwin J, Chothia C. 1979. Haemoglobin: the structural changes related to ligand binding and its allosteric mechanism. J Mol Biol 129:175-220. doi:10.1016/0022-2836(79)90277-8

Bausewein T, Mills DJ, Langer JD, Nitschke B, Nussberger S, Kühlbrandt W. 2017. Cryo-EM structure of the TOM core complex from Neurospora crassa. Cell 170:693-700. doi:10.1016/j.cell.2017.07.012

Bausewein T, Naveed H, Liang J, Nussberger S. 2020. The structure of the TOM core complex in the mitochondrial outer membrane. Biol Chem 401:687-697. doi:10.1515/hsz-2020-0104 
Becker L, Bannwarth M, Meisinger C, Hill K, Model K, Krimmer T, Casadio R, Truscott KN, Schulz GE, Pfanner N, Wagner R. 2005. Preprotein translocase of the outer mitochondrial membrane: reconstituted Tom40 forms a characteristic TOM Pore. J Mol Biol 353:1011-1020. doi:10.1016/j.jmb.2005.09.019

Becker T, Song J, Pfanner N. 2019. Versatility of preprotein transfer from the cytosol to mitochondria. Trends Cell Biol 29:534-548. doi:10.1016/j.tcb.2019.03.007

Benz R. 2006. Bacterial and Eukaryotic Porins: Structure, Function, Mechanism. John Wiley \& Sons.

Benz R, Kottke M, Brdiczka D. 1990. The cationically selective state of the mitochondrial outer membrane pore: a study with intact mitochondria and reconstituted mitochondrial porin. Biochim Biophys Acta - Biomembr 1022:311-318. doi:10.1016/0005-2736(90)90279-W

Bieligmeyer M, Artukovic F, Nussberger S, Hirth T, Schiestel T, Müller M. 2016. Reconstitution of the membrane protein OmpF into biomimetic block copolymer-phospholipid hybrid membranes. Beilstein J Nanotechnol 7:881-892. doi:10.3762/bjnano.7.80

Brohawn SG, Campbell EB, MacKinnon R. 2014a. Physical mechanism for gating and mechanosensitivity of the human TRAAK $\mathrm{K}+$ channel. Nature 516:126-130. doi:10.1038/nature14013

Brohawn SG, Su Z, MacKinnon R. 2014b. Mechanosensitivity is mediated directly by the lipid membrane in TRAAK and TREK1 K+ channels. Proc Natl Acad Sci 111:3614-3619. doi:10.1073/pnas.1320768111

Callegari S, Cruz-Zaragoza LD, Rehling P. 2020. From TOM to the TIM23 complex - handing over of a precursor. Biol Chem 401:709-721. doi:10.1515/hsz-2020-0101

Chacinska A, Lind M, Frazier AE, Dudek J, Meisinger C, Geissler A, Sickmann A, Meyer HE, Truscott KN, Guiard B, Pfanner N, Rehling P. 2005. Mitochondrial presequence translocase: switching between TOM tethering and motor recruitment involves Tim21 and Tim17. Cell 120:817-829. doi:10.1016/j.cell.2005.01.011

Davis MM, Lamichhane R, Bruce BD. 2021. Elucidating protein translocon dynamics with singlemolecule precision. Trends Cell Biol 31:569-583. doi:10.1016/j.tcb.2021.03.009

Demuro A, Parker I. 2005. Optical patch-clamping: single-channel recording by imaging Ca2+ flux through individual muscle acetylcholine receptor channels. J Gen Physiol 126:179-192. doi:10.1085/jgp.200509331

Deng Z, Maksaev G, Schlegel AM, Zhang J, Rau M, Fitzpatrick JAJ, Haswell ES, Yuan P. 2020. Structural mechanism for gating of a eukaryotic mechanosensitive channel of small conductance. Nat Commun 11:3690. doi:10.1038/s41467-020-17538-1

Donzeau M, Káldi K, Adam A, Paschen S, Wanner G, Guiard B, Bauer MF, Neupert W, Brunner M. 2000. Tim23 links the inner and outer mitochondrial membranes. Cell 101:401-412. doi:10.1016/S0092-8674(00)80850-8

Duncan AL, Reddy T, Kolds $\varnothing$ H, Hélie J, Fowler PW, Chavent M, Sansom MSP. 2017. Protein crowding and lipid complexity influence the nanoscale dynamic organization of ion channels in cell membranes. Sci Rep 7:16647. doi:10.1038/s41598-017-16865-6

Finney DJ. 1941. On the distribution of a variate whose logarithm is normally distributed. Suppl J R Stat Soc 7:155-161. doi:10.2307/2983663

Fujiwara TK, Iwasawa K, Kalay Z, Tsunoyama TA, Watanabe Y, Umemura YM, Murakoshi H, Suzuki KGN, Nemoto YL, Morone N, Kusumi A. 2016. Confined diffusion of transmembrane proteins and lipids induced by the same actin meshwork lining the plasma membrane. Mol Biol Cell 27:1101-1119. doi:10.1091/mbc.E15-04-0186

Ge J, Elferich J, Goehring A, Zhao H, Schuck P, Gouaux E. 2018. Structure of mouse protocadherin 15 of the stereocilia tip link in complex with LHFPL5. eLife 7:e38770. doi:10.7554/eLife.38770

Gevorkyan-Airapetov L, Zohary K, Popov-Čeleketić D, Mapa K, Hell K, Neupert W, Azem A, Mokranjac D. 2009. Interaction of Tim23 with Tim50 is essential for protein translocation by the mitochondrial TIM23 complex. J Biol Chem 284:4865-4872. doi:10.1074/jbc.M807041200 
Gold VA, Chroscicki P, Bragoszewski P, Chacinska A. 2017. Visualization of cytosolic ribosomes on the surface of mitochondria by electron cryo-tomography. EMBO Rep 18:1786-1800. doi:10.15252/embr.201744261

Gold VAM, Ieva R, Walter A, Pfanner N, van der Laan M, Kühlbrandt W. 2014. Visualizing active membrane protein complexes by electron cryotomography. Nat Commun 5:4129. doi:10.1038/ncomms5129

Haswell ES, Phillips R, Rees DC. 2011. Mechanosensitive Channels: What Can They Do and How Do They Do It? Structure 19:1356-1369. doi:10.1016/j.str.2011.09.005

Hill K, Model K, Ryan MT, Dietmeier K, Martin F, Wagner R, Pfanner N. 1998. Tom40 forms the hydrophilic channel of the mitochondrial import pore for preproteins. Nature 395:516-521. doi: $10.1038 / 26780$

Huang S, Romero-Ruiz M, Castell OK, Bayley H, Wallace MI. 2015. High-throughput optical sensing of nucleic acids in a nanopore array. Nat Nanotechnol 10:986-991. doi:10.1038/nnano.2015.189

Jacobson K, Liu P, Lagerholm BC. 2019. The lateral organization and mobility of plasma membrane components. Cell 177:806-819. doi:10.1016/j.cell.2019.04.018

Jin P, Jan LY, Jan Y-N. 2020. Mechanosensitive ion channels: structural features relevant to mechanotransduction mechanisms. Annu Rev Neurosci 43:207-229. doi:10.1146/annurev-neuro070918-050509

Joo C, Ha T. 2008. Single-molecule FRET with total internal reflection microscopy In: Selvin PR, Ha T, editors. Single-Molecule Techniques: A Laboratory Manual. Cold Spring Harbor Laboratory Press. pp. 3-36.

Kefauver JM, Ward AB, Patapoutian A. 2020. Discoveries in structure and physiology of mechanically activated ion channels. Nature 587:567-576. doi:10.1038/s41586-020-2933-1

Kiessling V, Yang S-T, Tamm LK. 2015. Supported lipid bilayers as models for studying membrane domains In: Kenworthy AK, editor. Current Topics in Membranes. Academic Press. pp. 1-23. doi:10.1016/bs.ctm.2015.03.001

Koppel DE, Sheetz MP, Schindler M. 1981. Matrix control of protein diffusion in biological membranes. Proc Natl Acad Sci 78:3576-3580. doi:10.1073/pnas.78.6.3576

Künkele K-P, Heins S, Dembowski M, Nargang FE, Benz R, Thieffry M, Walz J, Lill R, Nussberger S, Neupert W. 1998. The preprotein translocation channel of the outer membrane of mitochondria. Cell 93:1009-1019. doi:10.1016/S0092-8674(00)81206-4

Kusumi A, Fujiwara TK, Chadda R, Xie M, Tsunoyama TA, Kalay Z, Kasai RS, Suzuki KGN. 2012. Dynamic organizing principles of the plasma membrane that regulate signal transduction: commemorating the fortieth anniversary of Singer and Nicolson's fluid-mosaic model. Annu Rev Cell Dev Biol 28:215-250. doi:10.1146/annurev-cellbio-100809-151736

Kuszak AJ, Jacobs D, Gurnev PA, Shiota T, Louis JM, Lithgow T, Bezrukov SM, Rostovtseva TK, Buchanan SK. 2015. Evidence of distinct channel conformations and substrate binding affinities for the mitochondrial outer membrane protein translocase pore Tom40. J Biol Chem 290:2620426217. doi:10.1074/jbc.M115.642173

Kuzmenko A, Tankov S, English BP, Tarassov I, Tenson T, Kamenski P, Elf J, Hauryliuk V. 2011. Single molecule tracking fluorescence microscopy in mitochondria reveals highly dynamic but confined movement of Tom40. Sci Rep 1:195. doi:10.1038/srep00195

Lee CP, Maksaev G, Jensen GS, Murcha MW, Wilson ME, Fricker M, Hell R, Haswell ES, Millar AH, Sweetlove L. 2016. MSL1 is a mechanosensitive ion channel that dissipates mitochondrial membrane potential and maintains redox homeostasis in mitochondria during abiotic stress. Plant J Cell Mol Biol 88:809-825. doi:10.1111/tpj.13301

Lemeshko SV, Lemeshko VV. 2000. Metabolically derived potential on the outer membrane of mitochondria: a computational model. Biophys $J$ 79:2785-2800. doi:10.1016/S00063495(00)76518-0 
Lemeshko VV. 2002. Model of the outer membrane potential generation by the inner membrane of mitochondria. Biophys J 82:684-692. doi:10.1016/S0006-3495(02)75431-3

Leptihn S, Castell OK, Cronin B, Lee E-H, Gross LCM, Marshall DP, Thompson JR, Holden M, Wallace MI. 2013. Constructing droplet interface bilayers from the contact of aqueous droplets in oil. Nat Protoc 8:1048-1057. doi:10.1038/nprot.2013.061

Li Y, Hu Y, Wang J, Liu X, Zhang W, Sun L. 2020. Structural insights into a plant mechanosensitive ion channel MSL1. Cell Rep 30:4518-4527.e3. doi:10.1016/j.celrep.2020.03.026

Mager F, Gessmann D, Nussberger S, Zeth K. 2011. Functional refolding and characterization of two Tom40 isoforms from human mitochondria. J Membr Biol 242:11-21. doi:10.1007/s00232-0119372-8

Mahendran KR, Lamichhane U, Romero-Ruiz M, Nussberger S, Winterhalter M. 2013. Polypeptide translocation through the mitochondrial TOM channel: temperature-dependent rates at the singlemolecule level. J Phys Chem Lett 4:78-82. doi:10.1021/jz301790h

Martinac B. 2004. Mechanosensitive ion channels: molecules of mechanotransduction. J Cell Sci 117:2449-2460. doi:10.1242/jcs.01232

Mokranjac D, Sichting M, Popov-Čeleketić D, Mapa K, Gevorkyan-Airapetov L, Zohary K, Hell K, Azem A, Neupert W. 2009. Role of Tim50 in the transfer of precursor proteins from the outer to the inner membrane of mitochondria. Mol Biol Cell 20:1400-1407. doi:10.1091/mbc.e08-09-0934

Namba T. 2019. BAP31 regulates mitochondrial function via interaction with Tom40 within ERmitochondria contact sites. Sci Adv 5:eaaw1386. doi:10.1126/sciadv.aaw1386

Nawrocki G, Im W, Sugita Y, Feig M. 2019. Clustering and dynamics of crowded proteins near membranes and their influence on membrane bending. Proc Natl Acad Sci 116:24562-24567. doi:10.1073/pnas.1910771116

Palmer CS, Lou J, Kouskousis B, Pandzic E, Anderson AJ, Kang Y, Hinde E, Stojanovski D. 2021. Superresolution microscopy reveals the arrangement of inner membrane protein complexes in mammalian mitochondria. J Cell Sci 134:1-11.

Pfanner N, Warscheid B, Wiedemann N. 2019. Mitochondrial protein organization: from biogenesis to networks and function. Nat Rev Mol Cell Biol 20:267-284. doi:10.1038/s41580-018-0092-0

Pliotas C, Naismith JH. 2017. Spectator no more, the role of the membrane in regulating ion channel function. Curr Opin Struct Biol 45:59-66. doi:10.1016/j.sbi.2016.10.017

Poynor M, Eckert R, Nussberger S. 2008. Dynamics of the preprotein translocation channel of the outer membrane of mitochondria. Biophys J 95:1511-1522. doi:10.1529/biophysj.108.131003

Ramadurai S, Holt A, Krasnikov V, van den Bogaart G, Killian JA, Poolman B. 2009. Lateral diffusion of membrane proteins. J Am Chem Soc 131:12650-12656. doi:10.1021/ja902853g

Rapaport D, Künkele K-P, Dembowski M, Ahting U, Nargang FE, Neupert W, Lill R. 1998. Dynamics of the TOM complex of mitochondria during binding and translocation of preproteins. Mol Cell Biol 18:5256-5262. doi:10.1128/MCB.18.9.5256

Romero-Ruiz M, Mahendran KR, Eckert R, Winterhalter M, Nussberger S. 2010. Interactions of mitochondrial presequence peptides with the mitochondrial outer membrane preprotein translocase TOM. Biophys J 99:774-781. doi:10.1016/j.bpj.2010.05.010

Sackmann E. 1996. Supported membranes: scientific and practical applications. Science 271:43-48. doi:10.1126/science.271.5245.43

Schmidt O, Harbauer AB, Rao S, Eyrich B, Zahedi RP, Stojanovski D, Schönfisch B, Guiard B, Sickmann A, Pfanner N, Meisinger C. 2011. Regulation of mitochondrial protein import by cytosolic kinases. Cell 144:227-239. doi:10.1016/j.cell.2010.12.015

Scorrano L, De Matteis MA, Emr S, Giordano F, Hajnóczky G, Kornmann B, Lackner LL, Levine TP, Pellegrini L, Reinisch K, Rizzuto R, Simmen T, Stenmark H, Ungermann C, Schuldiner M. 2019. Coming together to define membrane contact sites. Nat Commun 10:1287. doi:10.1038/s41467019-09253-3 
Shiota T, Imai K, Qiu J, Hewitt VL, Tan K, Shen H-H, Sakiyama N, Fukasawa Y, Hayat S, Kamiya M, Elofsson A, Tomii K, Horton P, Wiedemann N, Pfanner N, Lithgow T, Endo T. 2015. Molecular architecture of the active mitochondrial protein gate. Science 349:1544-1548. doi:10.1126/science.aac6428

Sukhorukov VM, Dikov D, Busch K, Strecker V, Wittig I, Bereiter-Hahn J. 2010. Determination of protein mobility in mitochondrial membranes of living cells. Biochim Biophys Acta - Biomembr 1798:2022-2032. doi:10.1016/j.bbamem.2010.07.016

Tanaka M, Sackmann E. 2005. Polymer-supported membranes as models of the cell surface. Nature 437:656-663. doi: $10.1038 /$ nature04164

Teng J, Loukin S, Anishkin A, Kung C. 2015. The force-from-lipid (FFL) principle of mechanosensitivity, at large and in elements. Pflüg Arch - Eur J Physiol 467:27-37. doi:10.1007/s00424-014-1530-2

Tucker K, Park E. 2019. Cryo-EM structure of the mitochondrial protein-import channel TOM complex at near-atomic resolution. Nat Struct Mol Biol 26:1158-1166. doi:10.1038/s41594-019-0339-2

van der Laan M, Wiedemann N, Mick DU, Guiard B, Rehling P, Pfanner N. 2006. A role for Tim21 in membrane-potential-dependent preprotein sorting in mitochondria. Curr Biol 16:2271-2276. doi:10.1016/j.cub.2006.10.025

van Wilpe S, Ryan MT, Hill K, Maarse AC, Meisinger C, Brix J, Dekker PJT, Moczko M, Wagner R, Meijer M, Guiard B, Hönlinger A, Pfanner N. 1999. Tom22 is a multifunctional organizer of the mitochondrial preprotein translocase. Nature 401:485-489. doi:10.1038/46802

Vogel F, Bornhövd C, Neupert W, Reichert AS. 2006. Dynamic subcompartmentalization of the mitochondrial inner membrane. J Cell Biol 175:237-247. doi:10.1083/jcb.200605138

Waegemann K, Popov-Čeleketić D, Neupert W, Azem A, Mokranjac D. 2015. Cooperation of TOM and TIM23 complexes during translocation of proteins into mitochondria. $J$ Mol Biol, Elucidation of Protein Translocation Pathways (Part I) 427:1075-1084. doi:10.1016/j.jmb.2014.07.015

Walewska A, Kulawiak B, Szewczyk A, Koprowski P. 2018. Mechanosensitivity of mitochondrial largeconductance calcium-activated potassium channels. Biochim Biophys Acta BBA - Bioenerg, 20th European Bioenergetics Conference 1859:797-805. doi:10.1016/j.bbabio.2018.05.006

Wang W, Chen X, Zhang L, Yi J, Ma Q, Yin J, Zhuo W, Gu J, Yang M. 2020. Atomic structure of human TOM core complex. Cell Discov 6:1-10. doi:10.1038/s41421-020-00198-2

Wang Yang, Guo Y, Liu C, Wang L, Zhang A, Yan Z, Song C. 2019. Push-to-open: the gating mechanism of the tethered mechanosensitive ion channel NompC. bioRxiv 853721. doi:10.1101/853721

Wang Y, Yan S, Zhang P, Zeng Z, Zhao D, Wang J, Chen H, Huang S. 2018. Osmosis-driven motiontype modulation of biological nanopores for parallel optical nucleic acid sensing. ACS Appl Mater Interfaces 10:7788-7797. doi:10.1021/acsami.7b18347

Wang Yuqin, Wang Yu, Du X, Yan S, Zhang P, Chen H-Y, Huang S. 2019. Electrode-free nanopore sensing by DiffusiOptoPhysiology. Sci Adv 5:eaar3309.

Wiedemann N, Pfanner N. 2017. Mitochondrial machineries for protein import and assembly. Аппи Rev Biochem 86:685-714. doi:10.1146/annurev-biochem-060815-014352

Wurm CA, Neumann D, Lauterbach MA, Harke B, Egner A, Hell SW, Jakobs S. 2011. Nanoscale distribution of mitochondrial import receptor Tom20 is adjusted to cellular conditions and exhibits an inner-cellular gradient. Proc Natl Acad Sci 108:13546-13551. doi:10.1073/pnas.1107553108

\section{Acknowledgments}

We thank Beate Nitschke for help with protein preparation, and Stephan Eisler and Ke Zhou for help with the TIRF microscopy. We thank Robin Ghosh, for stimulating discussion, and the Baden-Württemberg Foundation for funding (BiofMO-6, SN). 


\section{Author contributions:}

SN initiated and directed the study. SW fluorescently labeled proteins, collected and processed the TIRF data. MH and SW wrote the software used for data and statistical analysis. SW, MH and SN analyzed results. SN wrote the initial paper draft and secured funding. SW, MW, MH and SN edited and reviewed the draft. LF, SL and MW provided initial expertise.

\section{Competing interests:}

All authors declare that they have no competing interests. 


\section{Figures}

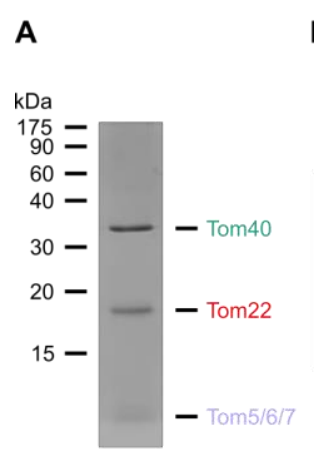

B

C
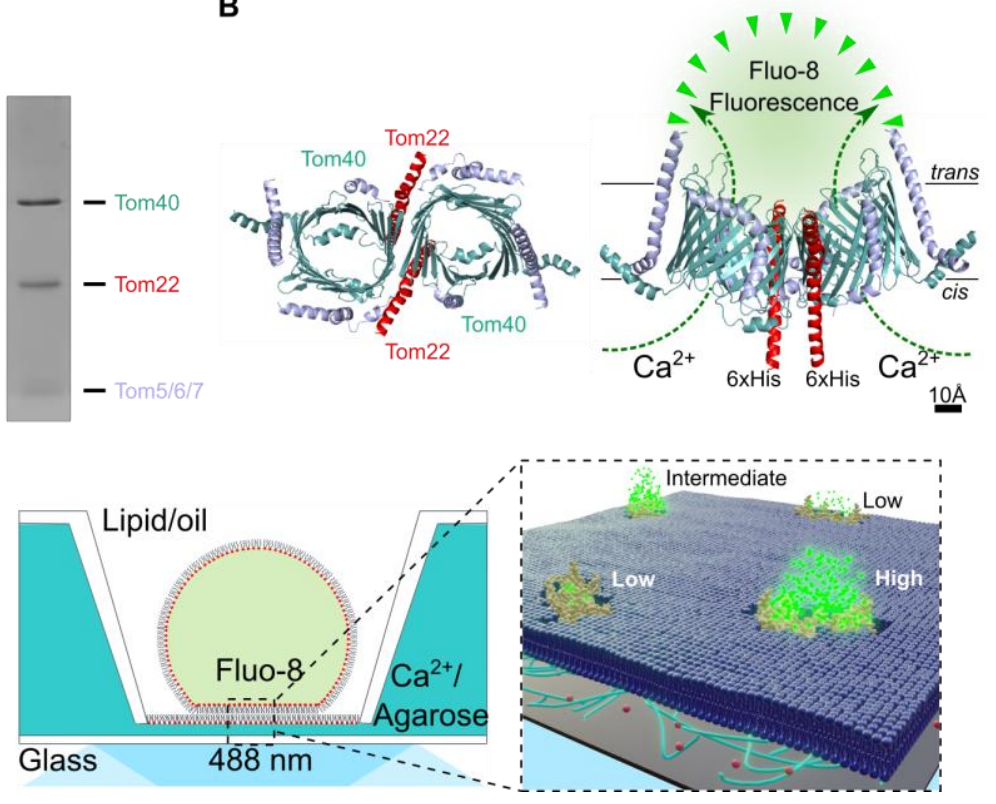

Fig. 1. Scheme for tracking single TOM-CC molecules and imaging their ion channel activity. (A) TOM-CC was isolated from mitochondria of a Neurospora strain carrying a Tom 22 with a hexahistidinyl tag (6xHis). Analysis of purified protein by SDS-polyacrylamide gel electrophoresis (SDS-PAGE) followed by Coomassie Blue staining revealed all known subunits of the core complex, Tom40, Tom22, Tom7, Tom6 and Tom5. The small subunits Tom7, Tom6 and Tom5 are not separated by SDS-PAGE. (B) Atomic model based on the cryoEM map of $N$. crassa TOM core complex (EMDB, EMD-3761; (Bausewein et al., 2020, 2017)). The ionic pathway through the two aqueous $\beta$-barrel Tom 40 pores is used to optically study the open-closed channel activity of individual TOM-CCs. Left, cytosolic view; right, side view; cis, mitochondria intermembrane space; trans, cytosol. Tom7, Tom6 and Tom5 are not labeled for clarity. (C) Single molecule tracking and channel activity sensing of TOM-CC in DIB membranes using electrodefree optical single-channel recording (Ef-oSCR). Left: Membranes are created through contact of lipid monolayer-coated aqueous droplets in a lipid/oil phase and a lipid monolayer on top of an agarose hydrogel. The cis side of the membrane contained $\mathrm{Ca}^{2+}$-ions, while having a $\mathrm{Ca}^{2+}$ sensitive fluorescent dye (Fluo-8) at the trans side. Right: $\mathrm{Ca}^{2+}$-ion flux through individual TOMCCs from cis to trans is driven by a $\mathrm{Ca}^{2+}$ concentration gradient, established around the two Tom 40 pores, and measured by monitoring Fluo- 8 emission in close proximity to the membrane using TIRF microscopy. Fluorescence signals reveal the local position of individual TOM-CCs, which is used to determine their mode of lateral diffusion in the membrane. The level of the fluorescence (high, intermediate and low intensity) correlates with corresponding permeability states of a TOM-CC molecule. A 100x TIRF objective is used both for illumination and imaging. Green dots, fluorescent Fluo-8; red dots, $\mathrm{Ca}^{2+}$ ions. 
A
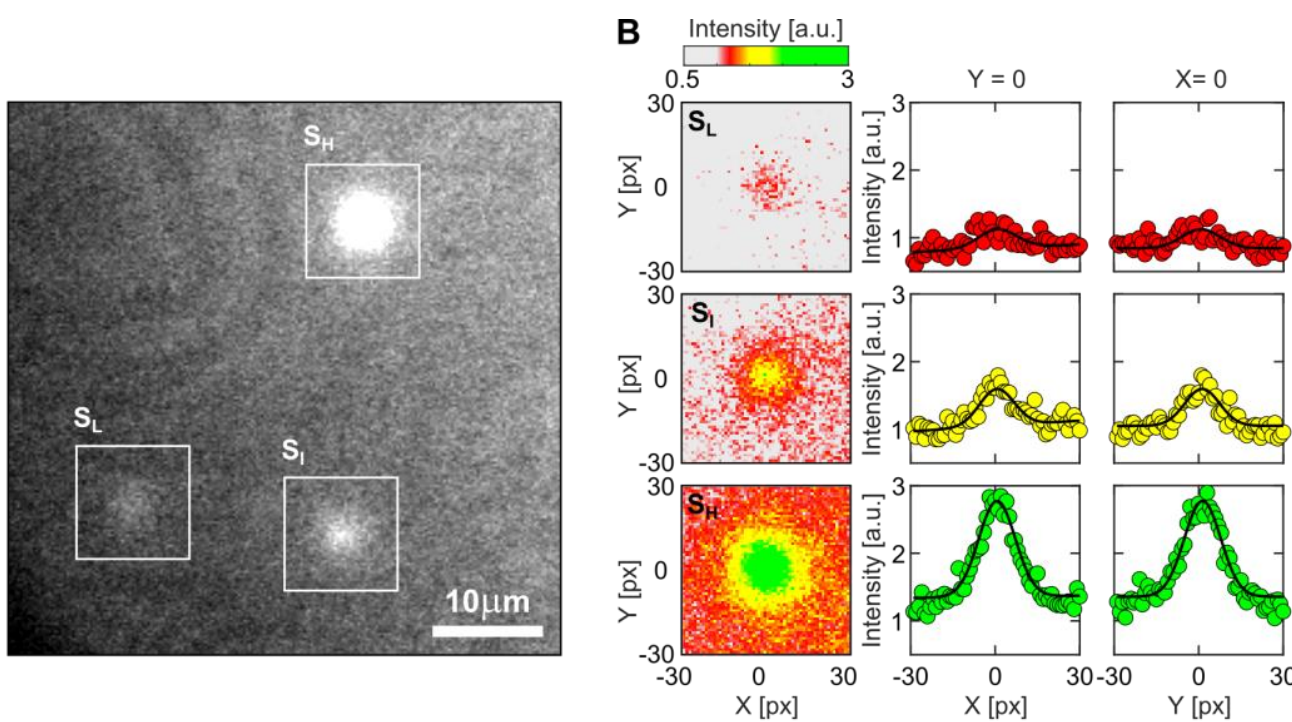

C

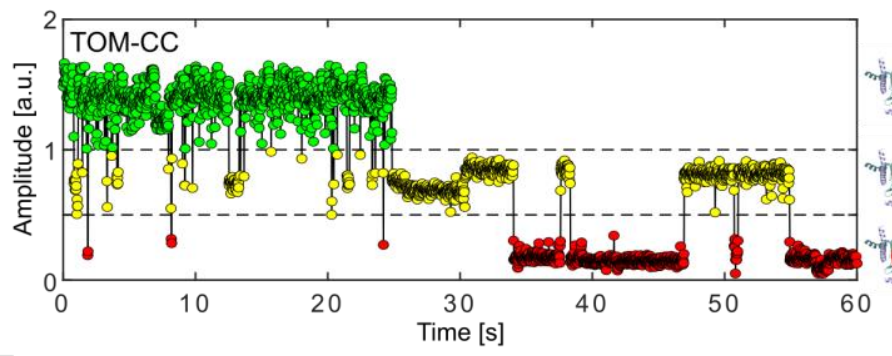

E

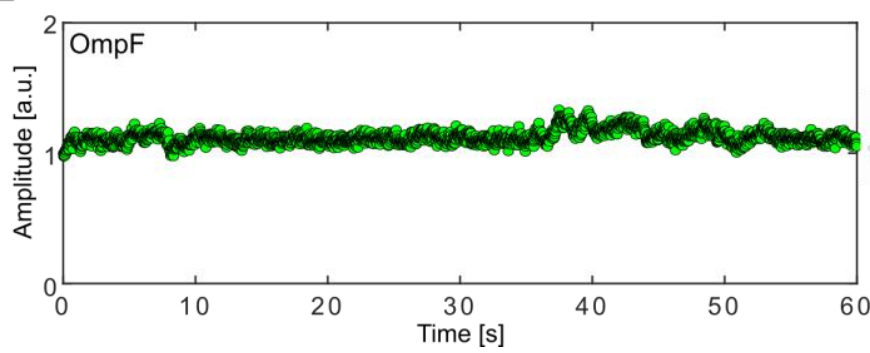

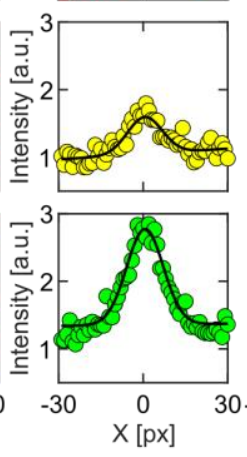

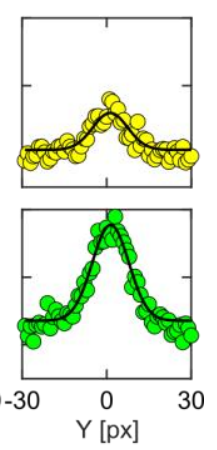

D

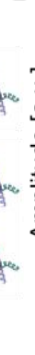

F
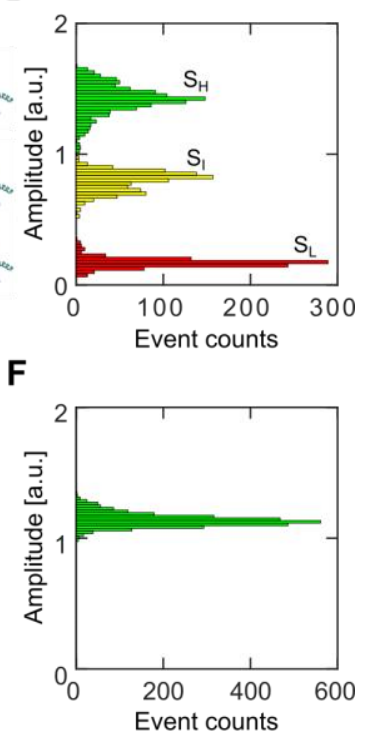

Fig. 2. Visualizing the two pore channel activity of TOM-CC. (A) Typical image $(N>5.3 \mathrm{x}$ $10^{5}$ ) of a non-modified agarose-supported DIB membrane with TOM-CC channels under $488 \mathrm{~nm}$ TIRF-illumination. The white squares mark spots of high $\left(\mathrm{S}_{\mathrm{H}}\right)$, intermediate $\left(\mathrm{S}_{\mathrm{I}}\right)$ and low $\left(\mathrm{S}_{\mathrm{L}}\right)$ intensity (Movie S1). (B) Fitting the fluorescence intensity profile of the three spots marked in (A) to two-dimensional Gaussian functions (Movie S2). Red, yellow and green intensity profiles represent TOM-CC in $\mathrm{S}_{\mathrm{L}}, \mathrm{S}_{\mathrm{I}}$ and $\mathrm{S}_{\mathrm{H}}$ demonstrating Tom40 channels, which are fully closed, one and two channels open, respectively. Pixel size, $0.16 \mu \mathrm{m}$. (C) Fluorescence amplitude trace and (D) amplitude histogram of the two-pore $\beta$-barrel protein channel TOM-CC. The TOM-CC channel switches between $\mathrm{S}_{\mathrm{H}}, \mathrm{S}_{\mathrm{I}}$, and $\mathrm{S}_{\mathrm{L}}$ permeability states over time. Inserts, schematic of $N$. crassa TOM core complex with two pores open in $\mathrm{S}_{\mathrm{H}}$ (top), one pore open in $\mathrm{S}_{\mathrm{I}}$ (middle), and two pores closed in $\mathrm{S}_{\mathrm{L}}$ (bottom) (EMDB, EMD-3761; (Bausewein et al., 2020, 2017)); (E) Representative single-channel optical recording and (F) amplitude histogram of the three-pore $\beta$ barrel protein OmpF used as a control, which is completely embedded in the lipid bilayer. In contrast to the two-pore $\beta$-barrel protein complex TOM-CC, OmpF exhibits only one permeability state over time. Insert, model of $E$. coli $\mathrm{OmpF}$ (PDB, 1OPF) with all three pores open. Data were acquired by Ef-oSCR as described in Fig.1C at a frame rate of $47.5 \mathrm{~s}^{-1}$. 
A

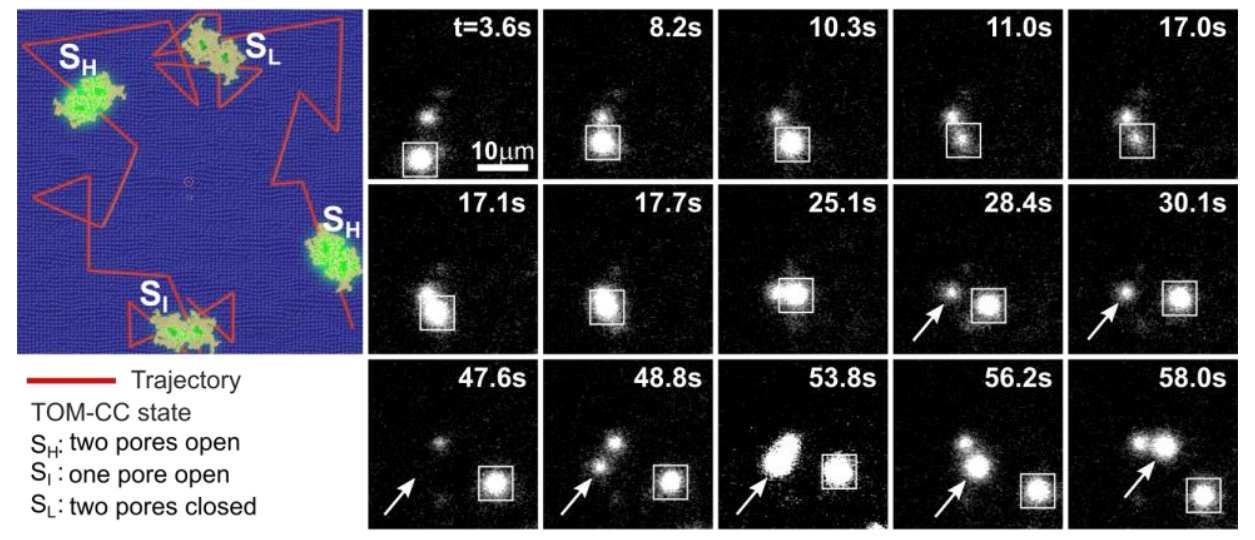

C

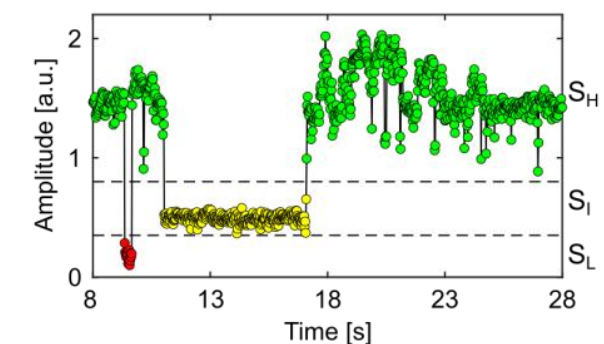

D

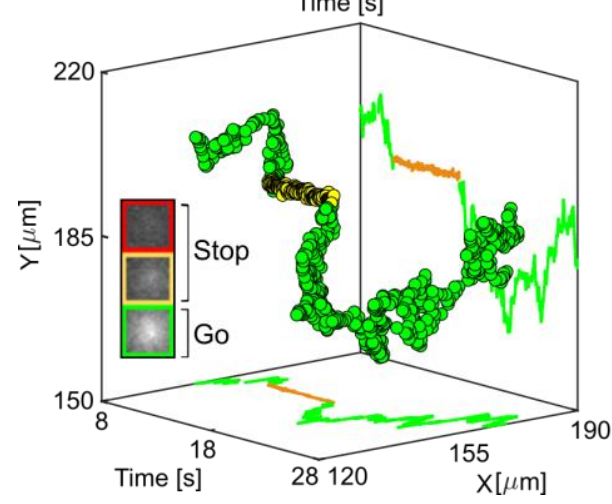

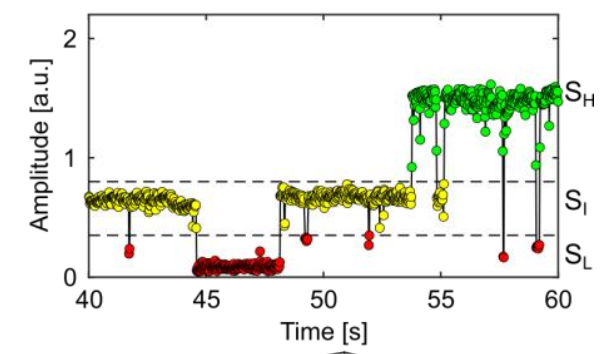

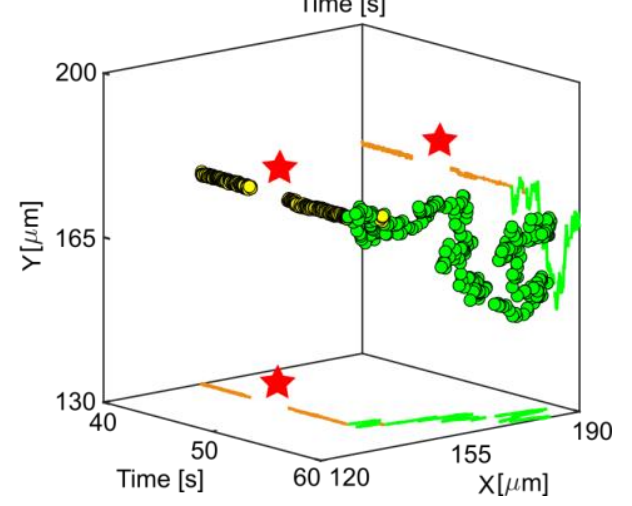

Fig. 3. Lateral mobility correlates with the channel activity of TOM-CC. (A) Scheme for imaging both position and channel activity of single TOM-CCs. (B) Representative TIRF microscopy images of a non-modified agarose-supported DIB membrane with three TOM-CC molecules taken from a time series of $60 \mathrm{~s}$. The square-marked spot displays lateral motion, interrupted by a transient arrest between $t=11.0 \mathrm{~s}$ and $t=17.0 \mathrm{~s}$. The arrow-marked spot corresponds to a non-moving TOM-CC until $t=48.8 \mathrm{~s}$. Afterwards, it starts moving. Both moving spots show high fluorescence intensity $\left(\mathrm{S}_{\mathrm{H}}\right)$; the non-moving spots display intermediate $\left(\mathrm{S}_{\mathrm{I}}\right)$ or dark $\left(\mathrm{S}_{\mathrm{L}}\right)$ fluorescence intensity (Movie $\mathrm{S} 3$ ). (C and D) Fluorescent amplitude trace and corresponding trajectory of the square- and arrow- marked TOM-CC as shown in (B) highlighted for two different time windows. Plots on top shows the change of amplitude over time, and plots on the bottom show the respective spatiotemporal dynamics for the three states. Comparison of the trajectories of single TOM-CC molecules with their corresponding amplitude traces reveals a direct correlation between stop-and-go movement and open-closed channel activity. Lateral diffusion of TOM-CCs in the DIB membrane is interrupted by temporary arrest, presumably due to transient linkage to the underlying agarose hydrogel. Although weak intensity profiles in $\mathrm{S}_{\mathrm{I}}$ do not allow accurate position determination, the fluorescent spots disappear and reappear at the same spatial $\mathrm{x}, \mathrm{y}$ coordinates (red stars). The higher amplitude (C, top) between $t=17.1 \mathrm{~s}$ and $t=25.1 \mathrm{~s}$ is due to the overlap between two adjacent spots. Green, TOM-CC is freely diffusive in $\mathrm{S}_{\mathrm{H}}$; yellow and red, immobile TOM-CC in $\mathrm{S}_{\mathrm{I}}$ and $\mathrm{S}_{\mathrm{L}}$. Data were acquired by Ef-oSCR as described in Fig.1C at a frame rate of $47.5 \mathrm{~s}^{-1}$. A total of $n_{\mathrm{TOM}}=64$ amplitude traces and trajectories were analyzed. 
A Low intensity

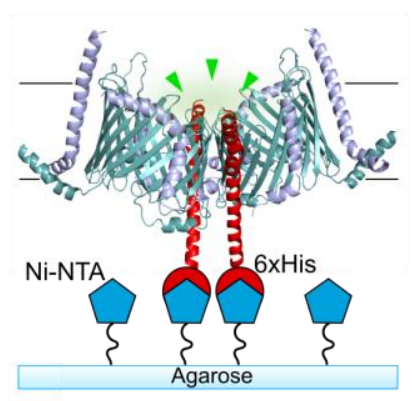

Non-diffusive mode

$\mathrm{S}_{\mathrm{L}}$ two pores closed
Intermediate intensity

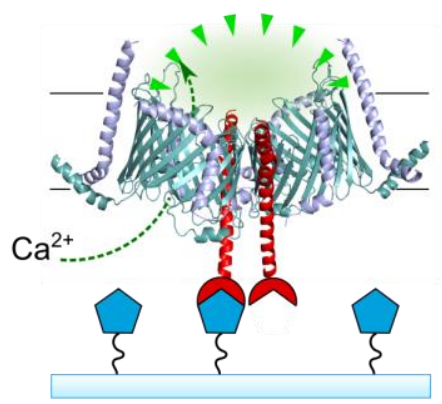

Non-diffusive mode $\mathrm{S}_{1}$ one pore open

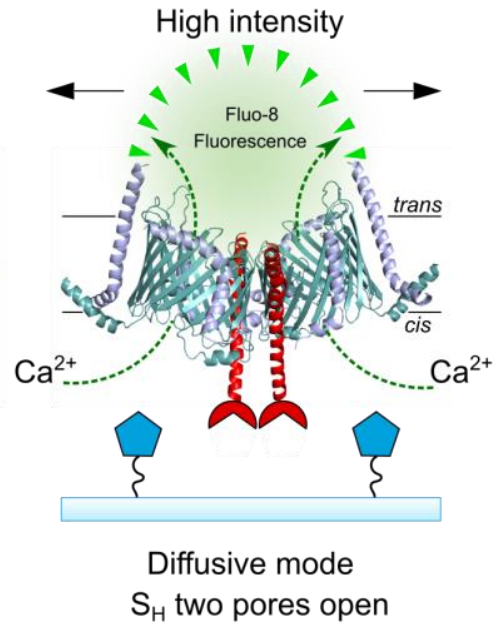

C
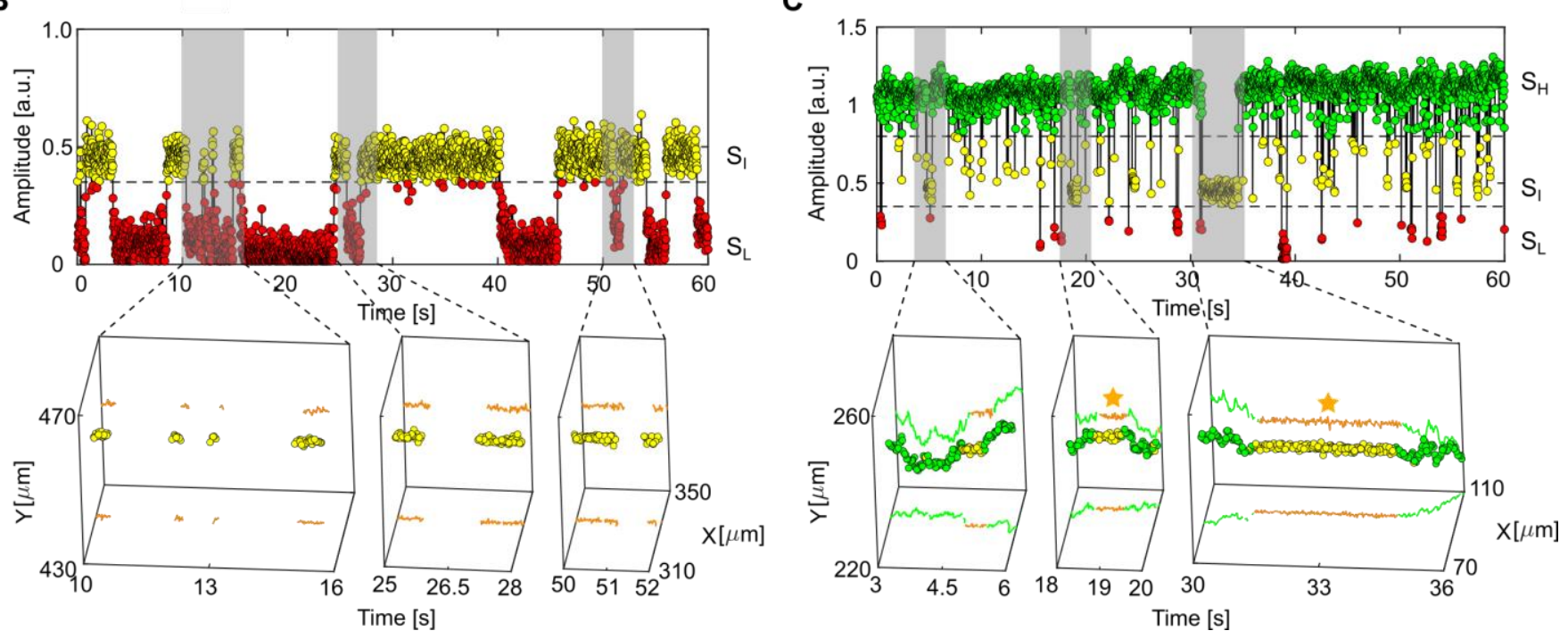

Fig. 4. Controlled immobilization of TOM-CC triggers channel closures. (A) Schematic representation of individual TOM-CC channels in DIB membranes supported by Ni-NTAmodified agarose. TOM-CC molecules can be permanently linked to the underlying hydrogel via His-tagged Tom22. Tethered and non-tethered TOM-CC molecules in closed $\left(\mathrm{S}_{\mathrm{I}}\right.$ and $\left.\mathrm{S}_{\mathrm{L}}\right)$ and open $\left(\mathrm{S}_{\mathrm{H}}\right)$ states are indicated, respectively. (B) Fluorescent amplitude trace (top) of a TOM-CC channel permanently tethered to Ni-NTA-modified agarose. The trajectory segments (bottom) correspond to the time periods of the amplitude traces marked in grey (Movie S6: bottom). Nondiffusive, permanently immobilized TOM-CC is only found in $\mathrm{S}_{\mathrm{I}}$ or $\mathrm{S}_{\mathrm{L}}$, indicating that tight binding of the His-tagged Tom22 domain (Fig. 1B) to Ni-NTA-modified agarose triggers closure of the $\beta$-barrel TOM-CC pores. (C) Fluorescence amplitude trace (top) of a TOM-CC channel transiently and non-specifically entangled by Ni-NTA-modified agarose. The trajectory segments (bottom) correspond to the time periods of the amplitude traces marked in grey (Movie S6 bottom). The movement of TOM-CC is interrupted twice at the same spatial $x, y$ membrane position from $t_{1}=18.56 \mathrm{~s}$ to $t_{2}=19.19 \mathrm{~s}$ and from $t_{3}=31.14 \mathrm{~s}$ to $t_{4}=34.55 \mathrm{~s}$ (yellow stars). Consistent with the data shown in Fig. 3, moving TOM-CC molecules in diffusive mode are found in the fully open $\mathrm{S}_{\mathrm{H}}$ state; transient tethering causes the TOM-CC $\beta$-barrels to close. Data were acquired by Ef-oSCR as described in Fig.1C at a frame rate of $47.5 \mathrm{~s}^{-1}$. A total of $n_{\mathrm{TOM}}=123$ amplitude traces and trajectories were analyzed. 


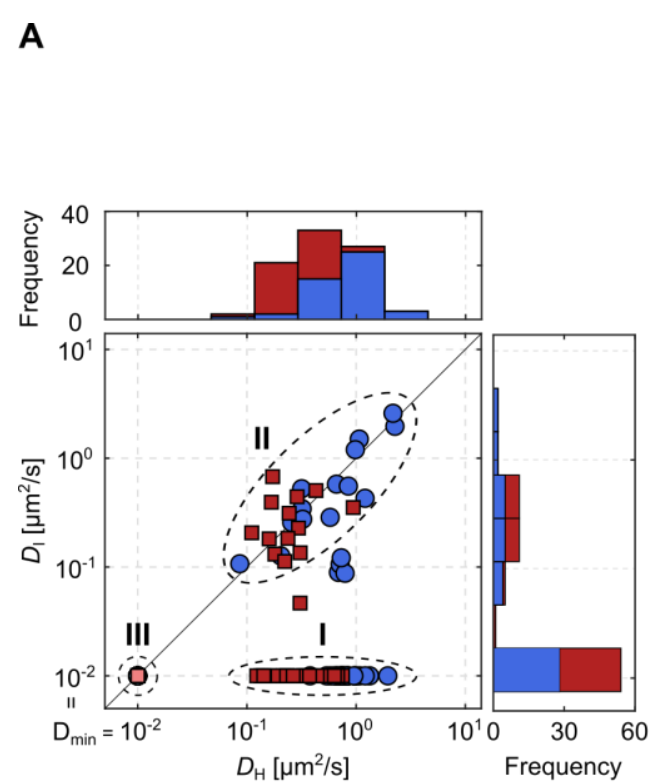

B

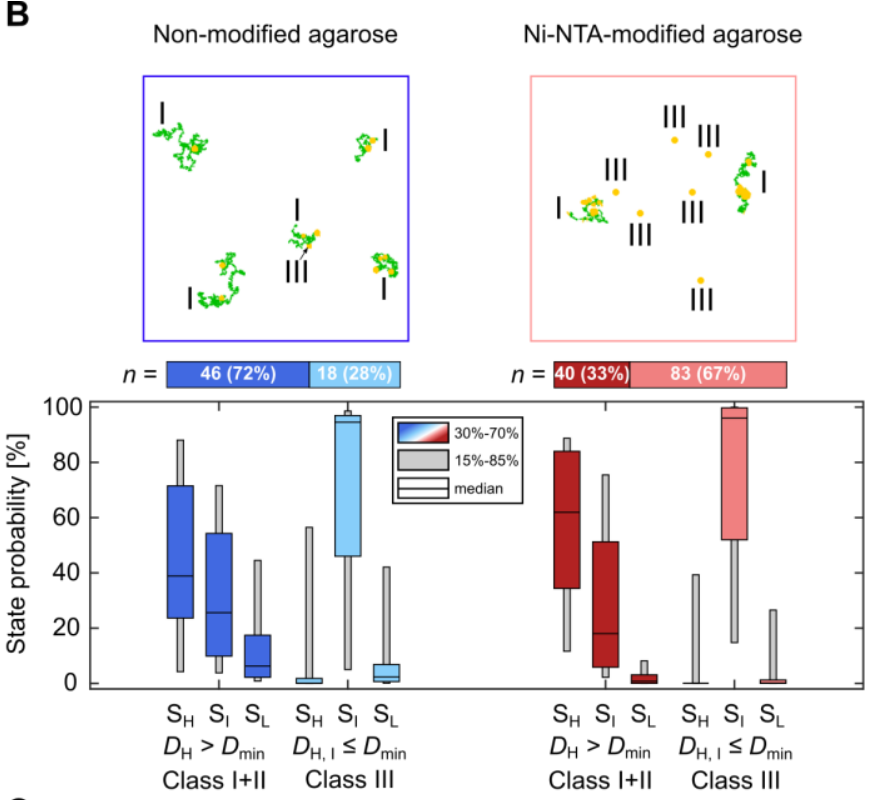

C

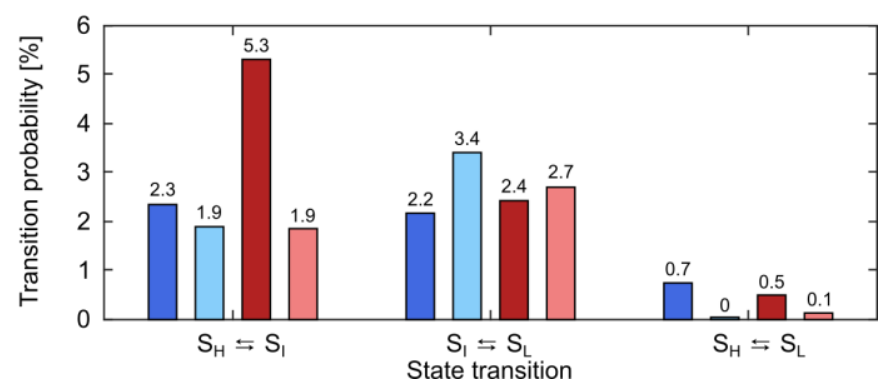

Fig. 5. Statistical correlation between channel activity and lateral mobility of TOM-CC. (A) $D_{\mathrm{I}}$ as a function of $D_{\mathrm{H}}$ individually plotted for all TOM-CC molecules in DIB membranes supported by non-modified (dark blue and light blue, $n=64$ ) and Ni-NTA modified agarose (dark red and light red, $n=123$ ). Frequency histograms of $D_{\mathrm{H}}$ and $D_{\mathrm{I}}$ are shown on top and right side, respectively. Three classes can be defined: a main class of moving particles in $\mathrm{S}_{\mathrm{H}}$ while being transiently tethered at $S_{I}$ (I), a second class of freely moving particles in $S_{H}$ and $S_{I}$ (II) and a third class of permanently tethered molecules in $S_{I}$ and $S_{L}$ (III). (B) Example trajectories (top and Movie S6) and state probabilities (bottom) of non-permanently $\left(D_{\mathrm{H}}>D_{\min }\right.$, class I+II) and permanently tethered $\left(D_{\mathrm{H}, \mathrm{I}} \leq D_{\mathrm{min}}\right.$, class III) TOM-CC in DIBs supported by non-modified and Ni-NTAmodified agarose. The probability of being in state $S_{\mathrm{H}}$ is higher for non-permanently $\left(D_{\mathrm{H}}>D_{\min }\right.$, class I+II, dark blue $[n=46]$ and dark red $[n=40])$ than for permanently tethered molecules $\left(D_{\mathrm{H}}\right.$, I $\leq D_{\min }$, class III, light blue $[n=18]$ and light red $\left.[n=83]\right)$. The probability of being in state $\mathrm{S}_{\mathrm{I}}$ is significantly higher for permanently $\left(D_{\mathrm{H}, \mathrm{I}} \leq D_{\min }\right.$, class III) than for non-permanently tethered particles $\left(D_{\mathrm{H}}>D_{\min }\right.$, class I+II). This suggests that binding of Tom 22 to Ni-NTA agarose below the membrane triggers closure of the TOM-CC channels. The data are represented as median; the confidence intervals are given between $15 \%$ to $85 \%$ and $30 \%$ to $70 \%$. Moving particles in $\mathrm{S}_{\mathrm{H}}$ are shown in the trajectories in green; transiently or permanently tethered molecules in $\mathrm{S}_{\mathrm{I}}$ are shown in yellow. (C) Absolute state transition probabilities classified by bidirectional state transitions as $\mathrm{S}_{\mathrm{H}} \leftrightarrows \mathrm{S}_{\mathrm{I}}, \mathrm{S}_{\mathrm{I}} \leftrightarrows \mathrm{S}_{\mathrm{L}}$, and $\mathrm{S}_{\mathrm{H}} \leftrightarrows \mathrm{S}_{\mathrm{L}}$. Diffusive TOM-CC molecules have a significantly higher transition probability for switching between $S_{H}$ and $S_{I}$ in DIBs supported by Ni-NTA-modified agarose $(\sim 5.3 \%)$ than in non-modified agarose membranes $(\sim 2.3 \%)$. This is consistent with the higher efficacy of TOM-CC-trapping by Ni-NTA-modified agarose compared to non-modified agarose. Classification of non-permanently and permanently tethered TOM-CC is shown at the left bottom. 


\section{Supplementary Materials}

\section{Correlation of mitochondrial TOM core complex stop-and-go and open-closed channel dynamics}

Shuo Wang, Lukas Findeisen, Sebastian Leptihn, Mark I. Wallace, Marcel Hörning*, Stephan Nussberger*

*Corresponding author. Email: Stephan.Nussberger@bio.uni-stuttgart.de, Marcel.Hoerning@bio.uni-stuttgart.de

\section{This PDF file includes:}

Figs. S1 to S5

Movie captions S1 to S6 

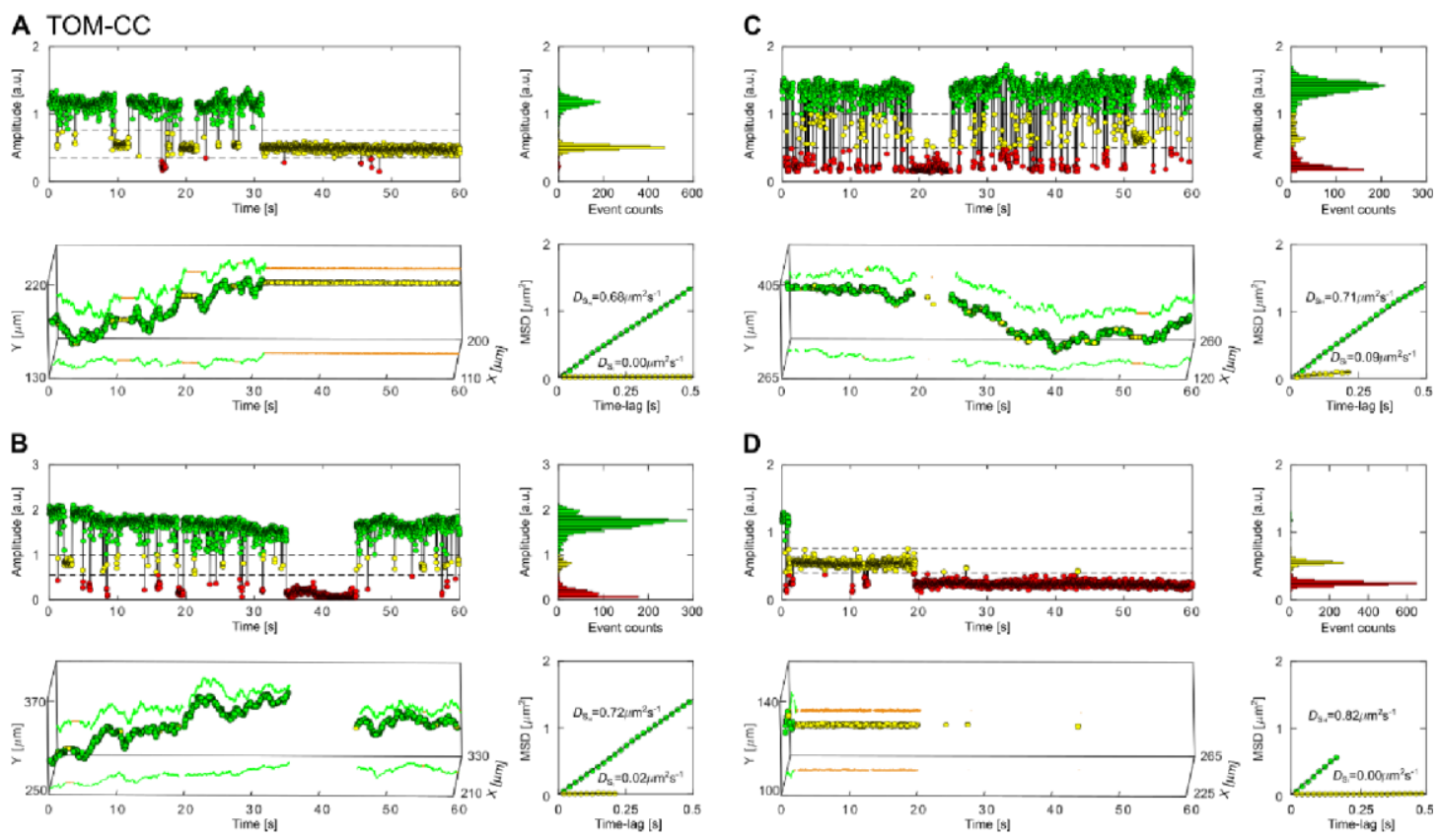

Fig. S1. Lateral mobility correlates with the open-closed channel activity of TOM-CC.

(A, B, C and D) Representative fluorescent amplitude traces of individual TOM-CC channels recorded in nonmodified agarose-supported DIB membranes. The amplitude recordings and trajectories indicate that the open-closed channel activity of TOM-CC correlates with the lateral membrane mobility of the complex. The fluorescent amplitude traces (upper left) and corresponding amplitude histograms (upper right) show three distinct ion permeation states $\left(\mathrm{S}_{\mathrm{H}}\right.$, green; $\mathrm{S}_{\mathrm{I}}$, yellow; $\mathrm{S}_{\mathrm{L}}$, red). The trajectories (bottom left) display two mobility states, moving (green) and non-moving (yellow). The mean square displacement (MSD, bottom right) increases linearly with time when TOM-CC is in $\mathrm{S}_{\mathrm{H}}$ state. The MSD does not change with time for TOM in the $S_{\text {I }}$ state. Due to limited resolution, trajectory points and MSD-plots are not shown for TOM$\mathrm{CC}$ in the low permeation state $\mathrm{S}_{\mathrm{L}}$. 


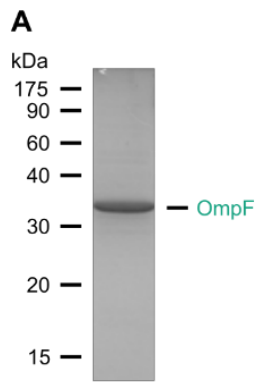

B
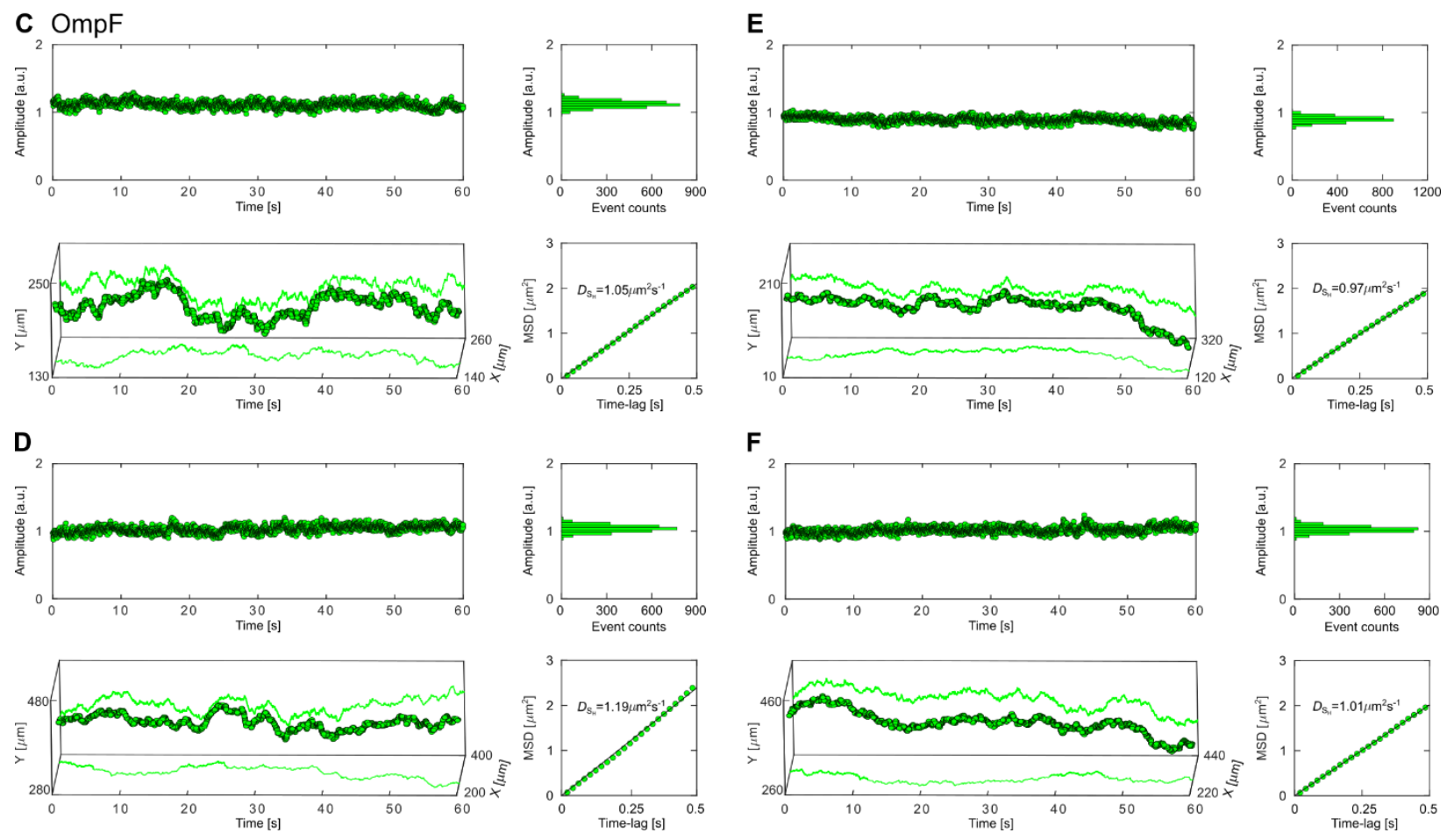

Fig. S2. Lateral mobility does not influence the open-closed channel activity of OmpF.

(A) SDS-PAGE of OmpF. The protein was purified from E. coli membranes by differential SDS extraction at $50^{\circ} \mathrm{C}$ and $37^{\circ} \mathrm{C}$, respectively. (B) Atomic model of OmpF (PDB, 1OPF). Left, view from the extracellular side; right, side view. $\mathrm{Ca}^{2+}$ flux through the three $\beta$-barrel pores is measured by electrode-free optical single channel recording (Ef-oSCR) using Fluo- 8 as $\mathrm{Ca}^{2+}$-sensitive dye, as described in Fig. 1. (C, D, E and F) Representative fluorescent amplitude traces of individual OmpF channels $(n=42)$ recorded in agarose-supported DIB membranes. The amplitude recordings and trajectories of the four examples indicate that the open-closed channel activity of OmpF does not correlate with the lateral mobility of the protein. OmpF displays only one ion permeation and lateral membrane mobility state, despite the fact that three channels are indicated in the atomic structure. 


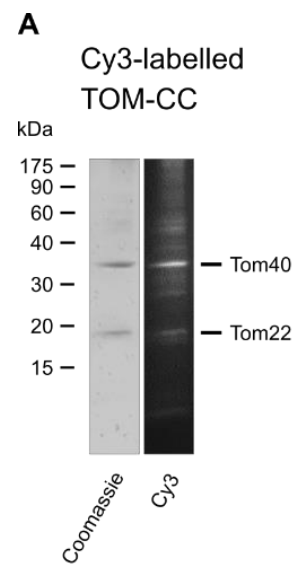

B

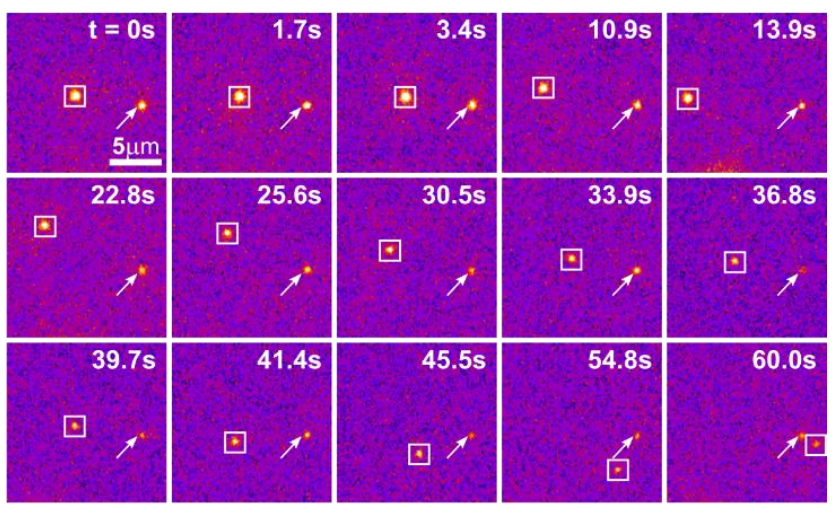

C

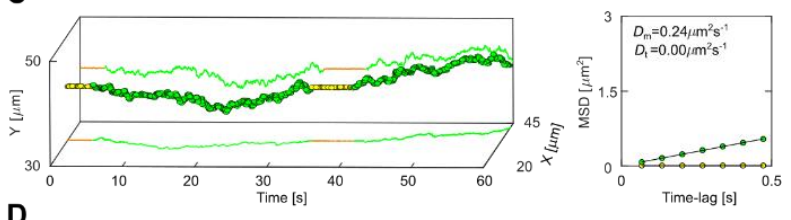

。

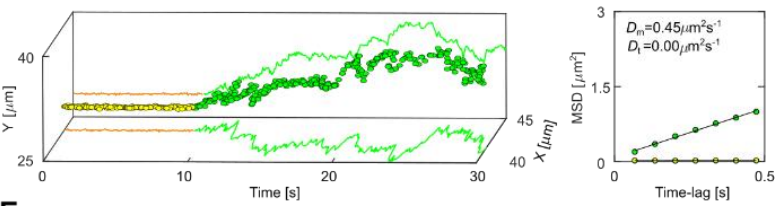

E

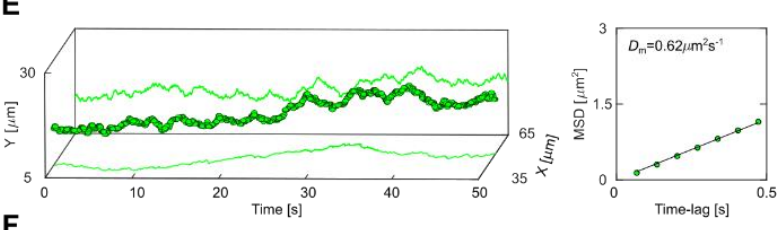

$\mathbf{F}$

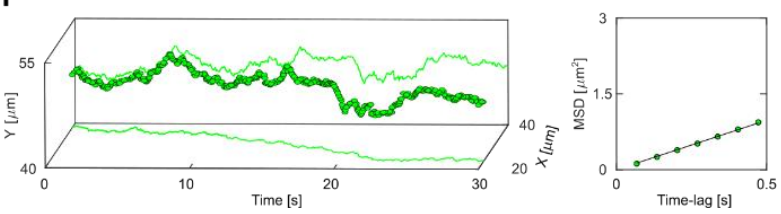

Fig. S3. Tracking fluorescent-labeled TOM-CC in non-modified agarose supported DIB membranes. (A) SDS-PAGE of Cy3-labeled TOM-CC analyzed by Coomassie Blue staining and Cy3 fluorescence, respectively. (B) Representative TIRF microscopy images of a DIB membrane with two Cy3-labeled TOM-CC molecules $(n=15)$ taken from a time series of $60 \mathrm{~s}$ (Movie S4). The square-marked spot displays lateral motion, interrupted by a transient arrest between $t=0 \mathrm{~s}$ and $t=3.3 \mathrm{~s}$, and between $t=33.9 \mathrm{~s}$ and $t=39.4 \mathrm{~s}$. The arrow-marked spot corresponds to a nonmoving TOM-CC. (C) Trajectory and diffusion coefficient of the squaremarked Cy3-labeled TOM-CC. (D, E and F) Trajectories and diffusion coefficients of additional Cy3-labeled TOMCCs. The color-coding of trajectories is the same as in Fig. 3. Note that the freely moving TOM$\mathrm{CC}$ molecule in Figures B and C stops at the same spatial x,y-position when it sweeps this position a second time, indicating a specific molecular trap or anchor point at this position below the membrane. 

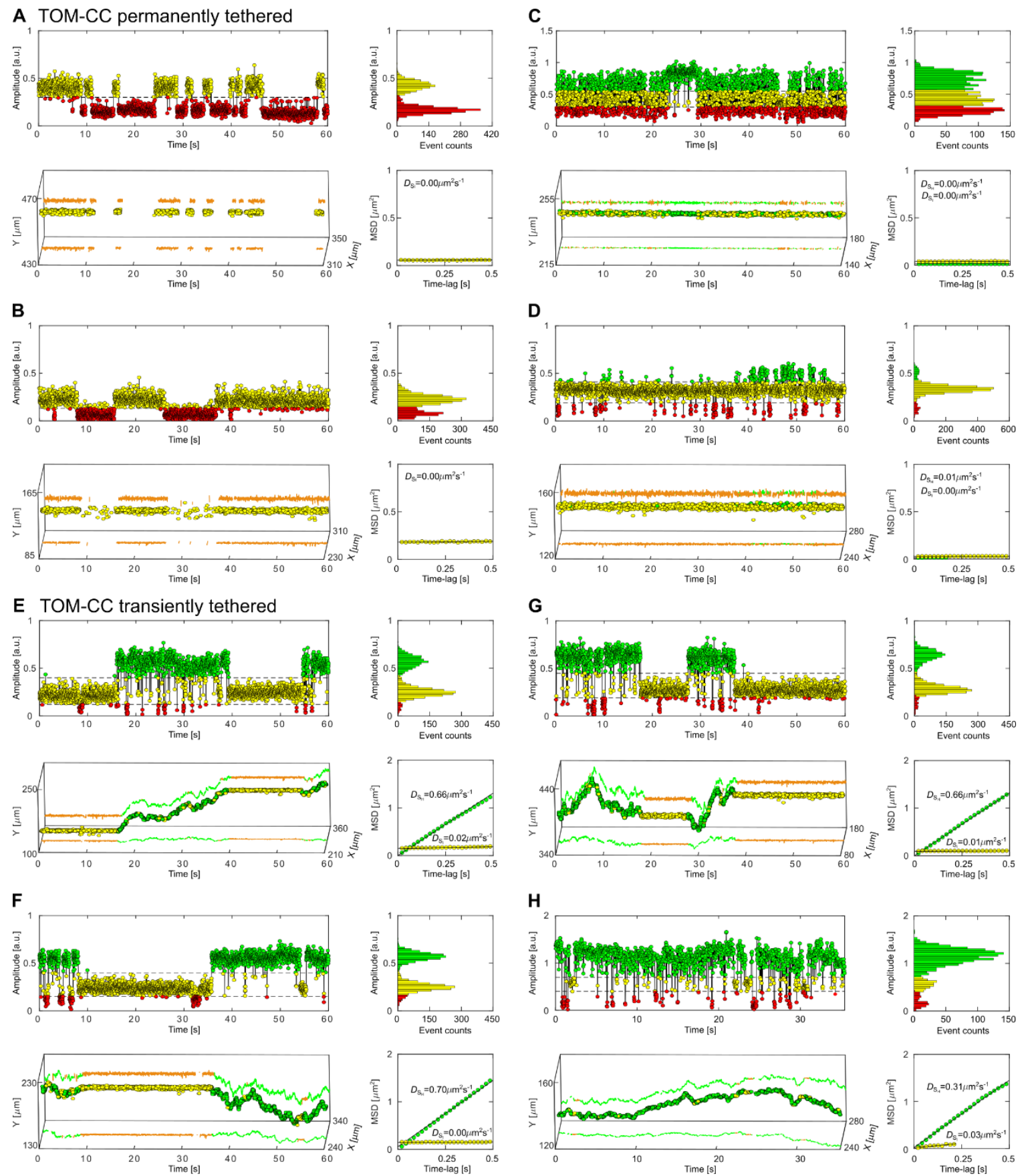

Fig. S4. Controlled immobilization of His-tagged TOM-CC triggers channel closures. Representative fluorescent amplitude traces of individual TOM-CC channels $(n=109)$ recorded in Ni-NTA-modified agarose-supported DIB membranes. (A, B, C and D) Particles are permanently trapped. (E, F, G and H) Particles are transiently trapped. Fluorescent amplitude traces (upper left), amplitude histograms (upper right), trajectories (bottom left) and mean square displacement plots (MSD, bottom right) are similar to those shown in Figs. 4B and 4C, respectively. The color-coding is the same as in Fig. 2. 

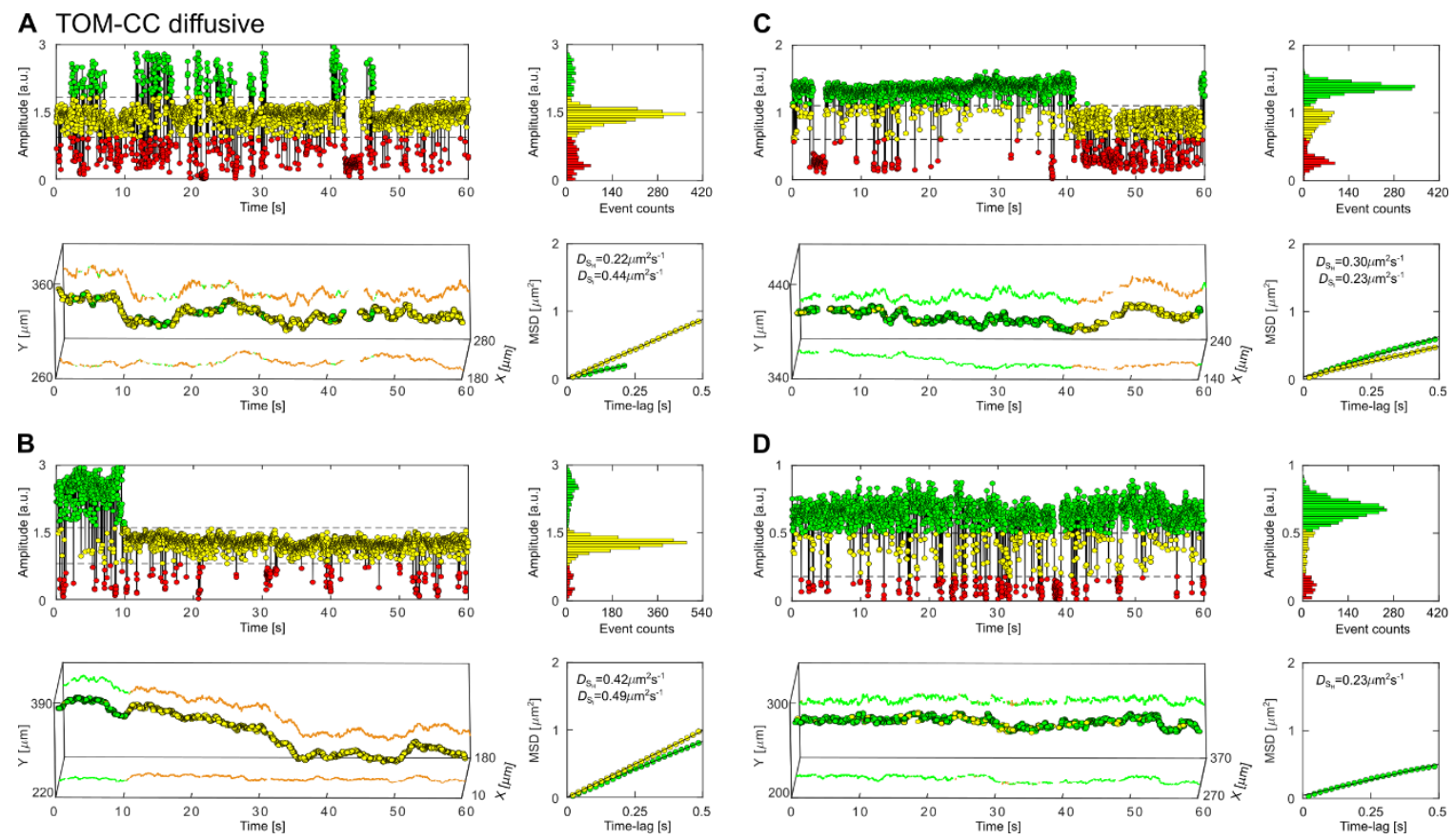

Fig. S5. Lateral mobility and open-closed channel activity of TOM-CC in Ni-NTA-modified agarose supported DIB membranes. (A, B, C and D) Fluorescent amplitude traces (upper left), amplitude histograms (upper right), trajectories (bottom left) and mean square displacement plots (MSD, bottom right) are shown. Particles $(n=14)$ are continuously moving and represent nontypical TOM-CCs. The color-coding is the same as in Fig. 2. 
Movie S1. Imaging the channel activity of single TOM-CC molecules, related to Fig. 2A. A $\mathrm{Ca}^{2+}$ indicator dye (Fluo-8) is used to monitor $\mathrm{Ca}^{2+}$-flux through individual TOM-CC channels in a non-modified agarose-supported DIB membrane using electrode-free optical single channel recording (Ef-oSCR). TOM-CCs appear as bright spots under $488 \mathrm{~nm}$ TIRF-illumination. The spots show high and intermediate intensity corresponding to two conformational states $\mathrm{S}_{\mathrm{H}}$ (green) and $S_{I}$ (yellow) with two pores and one pore open, respectively. The low intensity level represents a conformation $\mathrm{S}_{\mathrm{L}}$ (red) with both pores closed. Raw image data are shown; individual spots are marked according to their conformational states.

Movie S2. Time evolution of TOM-CC channel activity, related to Figs. 2B - 2C. Fitting the fluorescence intensity profiles of a single TOM-CC to two-dimensional Gaussian functions (right). Red, yellow and green intensity profiles represent TOM-CC in $\mathrm{S}_{\mathrm{L}}, \mathrm{S}_{\mathrm{I}}$ and $\mathrm{S}_{\mathrm{H}}$ demonstrating Tom 40 channels, which are fully closed, one, and two channels open, respectively. Original fluorescence intensities (left) were recorded at a pixel size of $0.16 \mu \mathrm{m}$ and at a frame rate of $47.51 \mathrm{~s}^{-1}$. No bleach correction and filter algorithm were applied.

Movie S3. Correlation between stop-and-go dynamics and open-closed channel activity of single TOM-CC molecules, related to Fig. 3. TIRF image recording (left) and trajectories (right) of individual TOM-CC molecules in a non-modified agarose supported DIB membrane. The square-marked spots display lateral motion (Go) interrupted by transient arrest (Stop). The red, yellow and green color coding corresponds to TOM-CC molecules, which are fully closed $\left(\mathrm{S}_{\mathrm{L}}\right)$, one $\left(\mathrm{S}_{\mathrm{I}}\right)$ and two $\left(\mathrm{S}_{\mathrm{H}}\right)$ channels open, respectively. Moving TOM-CC molecules in $\mathrm{S}_{\mathrm{H}}$ switch to $\mathrm{S}_{\mathrm{I}}$ or $S_{L}$ when they stop in the DIB membrane. Raw image data are shown; individual spots are marked according to their conformational states (left). The trajectories of moving TOM-CC molecules are colored in green; the trajectories of trapped TOM-CC molecules in $\mathrm{S}_{\mathrm{I}}$ are colored in yellow; the trajectories of trapped TOM-CC molecules in $\mathrm{S}_{\mathrm{L}}$ are not shown because weak intensity profiles do not allow accurate determination of the position of TOM-CC in the membrane plane.

Movie S4. Stop-and-go movement of fluorescently labeled TOM-CC, related to Fig. S3. TIRF image recording (left) and trajectories (right) of Cy3-labeled TOM-CC molecules in a nonmodified agarose supported DIB membrane. The square-marked spots display lateral motion (Go, green), interrupted by a transient arrest (Stop, yellow). Note that the freely moving TOMCC molecule stops at the same spatial $\mathrm{x}, \mathrm{y}$-position (yellow cross) when it crosses the same position a second time, indicating a specific molecular trap or anchor point at this position below the membrane. Raw image data are shown. Grey scales of individual images are transformed into pseudo colour images to better display movement of fluorescently labelled TOM-CC molecules. No bleach correction and filter algorithm were applied.

Movie S5. Lateral movement and channel activity of single OmpF molecules, related to Figs. 2E - 2F, and Fig. S2. TIRF image recording (left) and trajectories (right) of single OmpF molecules in a non-modified agarose supported DIB membrane. $\mathrm{A} \mathrm{Ca}^{2+}$ indicator dye (Fluo-8) is used to monitor $\mathrm{Ca}^{2+}$-flux through three-pore $\beta$-barrel OmpF channels using electrode-free optical single channel recording (Ef-oSCR). OmpF appears as bright spots under $488 \mathrm{~nm}$ TIRF illumination. The channel activity of $\mathrm{OmpF}$ does not correlate with the lateral mobility of the 
protein. OmpF displays only one ion permeation and lateral membrane mobility state. Raw image data are shown and individual spots are marked.

Movie S6. Single channel activity of transiently and permanently trapped TOM-CC molecules, related to Figs. 3, 4 and 5. TIRF image recordings (left) and trajectories (right) of single TOM-CC molecules in non-modified (top) and Ni-NTA-modified (bottom) agarose supported DIB membranes. The spots show high and intermediate intensity corresponding to two conformational states $S_{H}$ (green) and $S_{I}$ (yellow) with two pores and one pore open, respectively. The low intensity level represents a conformation $S_{L}($ red) with both pores closed. Diffusive TOM$\mathrm{CC}$ molecules are only in $\mathrm{S}_{\mathrm{H}}$ state. Non-diffusive molecules are either in $\mathrm{S}_{\mathrm{I}}$ or $\mathrm{S}_{\mathrm{L}}$. The permanently tethered fraction of TOM-CC in Ni-NTA-modified agarose is significantly larger compared to the fraction in non-modified agarose over time. Raw image data are shown; individual spots are marked according to their conformational states; trajectories of trapped TOM-CC molecules in $\mathrm{S}_{\mathrm{L}}$ are not shown because weak intensity profiles do not allow accurate determination of the position of TOM-CC in the membrane plane. Original fluorescence intensities were recorded at a pixel size of $0.16 \mu \mathrm{m}$ and at a frame rate of $47.51 \mathrm{~s}^{-1}$. 